\title{
Diet Induced Alteration of the Murine Intestinal Microbiome After Antibiotic Ablation
}

Adam Irvin

West Virginia University

Follow this and additional works at: https://researchrepository.wvu.edu/etd

\section{Recommended Citation}

Irvin, Adam, "Diet Induced Alteration of the Murine Intestinal Microbiome After Antibiotic Ablation" (2014). Graduate Theses, Dissertations, and Problem Reports. 598.

https://researchrepository.wvu.edu/etd/598

This Thesis is protected by copyright and/or related rights. It has been brought to you by the The Research Repository @ WVU with permission from the rights-holder(s). You are free to use this Thesis in any way that is permitted by the copyright and related rights legislation that applies to your use. For other uses you must obtain permission from the rights-holder(s) directly, unless additional rights are indicated by a Creative Commons license in the record and/ or on the work itself. This Thesis has been accepted for inclusion in WVU Graduate Theses, Dissertations, and Problem Reports collection by an authorized administrator of The Research Repository @ WVU. For more information, please contact researchrepository@mail.wvu.edu. 


\title{
Diet Induced Alteration of the Murine Intestinal Microbiome After Antibiotic Ablation
}

\author{
Adam Irvin \\ Thesis submitted \\ to the School of Medicine \\ at West Virginia University \\ Master of Science in \\ Biomedical Sciences \\ Christopher F. Cuff, Ph.D, Chair \\ Rosana Schafer, Ph.D \\ P. Roco LaSala, M.D.
}

in partial fulfillment of the requirements for the degree of

Department of Microbiology, Immunology and Cell Biology

Morgantown, WV

2014

Keywords: intestinal microbiome

Copyright 2014 Adam Irvin 


\title{
ABSTRACT \\ Diet Induced Alteration of the Murine Intestinal Microbiome After Antibiotic Ablation
}

\author{
Adam Irvin
}

\section{Background and Objective}

We detected a dominant non-cultivatable gram-positive coccobacillus associated with cultivatable yeast in feces of mice treated with a cocktail of broad-spectrum antibiotics while being fed a partially defined mouse chow that is commonly used for nutritional studies. The goal of this study was to determine the identity of the non-cultivatable grampositive coccobacillus.

\section{Methods}

Fecal samples were analyzed by Gram stain, quantitative flow cytometry, next generation sequencing of the V3 or V4 regions of the 16S rRNA gene, fluorescence microscopy, and sequence analysis of the tuf gene, which can distinguish among Streptococcus and Staphylococcus genera.

\section{$\underline{\text { Results }}$}

Fecal samples from antibiotic-treated mice displayed a ten-fold decline in bacterial cell number. High-throughput sequencing of the variable regions of the $16 \mathrm{~S}$ rRNA gene and tuf gene sequencing identified the major phylotype as Lactococcus. Lactococcus did not grow from fecal cultures, although Enterococcus casseliflavus did grow, which was a confounding finding. The mouse chow was heavily contaminated with non-viable Lactococcus. Appearance of intestinal yeast was dependent on the specific chow, although yeast was not detected in the chow.

\section{Conclusion}

Dead bacteria in food can modulate the intestinal microbiome. Whether and how food-derived dead bacteria alter intestinal physiology needs to be determined. 


\section{ACKNOWLEDGMENTS}

I would like to express my deepest appreciation to my advisor, Dr. Christopher F. Cuff, for his inspiration, guidance, support and understanding throughout my studies on this project. I would also like to express my appreciation to the other members of my committee, Dr. Rosana Schafer and Dr. P. Roco Lasala, for their assistance and thoughtful insight into my research. I would especially like to thank Dr. Andrew F. Cockburn for his assistance and expertise. 


\section{TABLE OF CONTENTS}

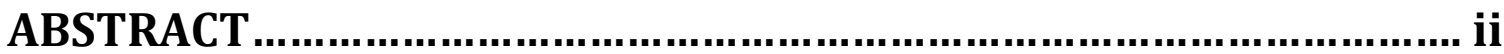

ACKNOWLEDGMENTS ................................................................. iii

TABLE OF CONTENTS ................................................................. iv

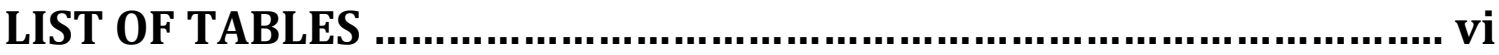

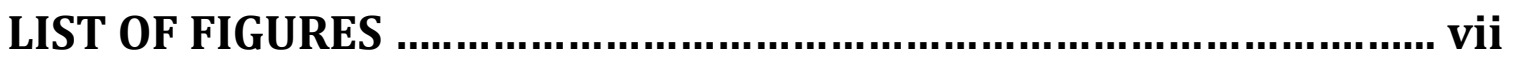

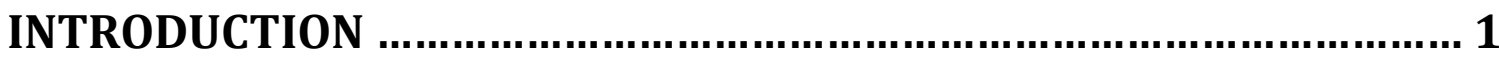

MATERIALS AND METHODS …................................................... 8

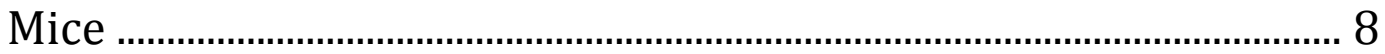

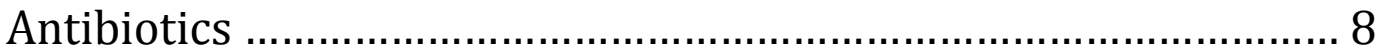

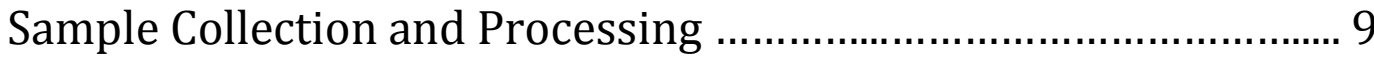

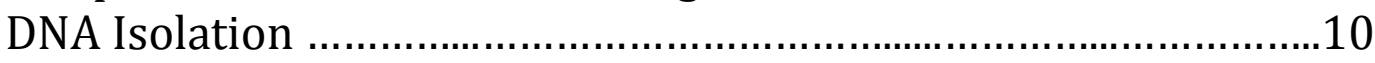

PCR and Fragment Purification ........................................................10

Illumina HiSeq High Throughput (HT) sequencing ..........................12

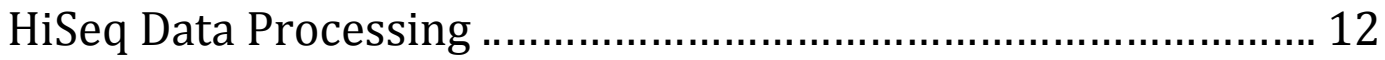

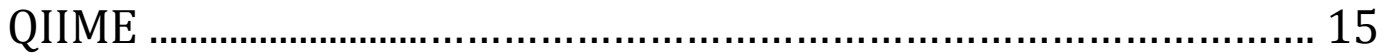

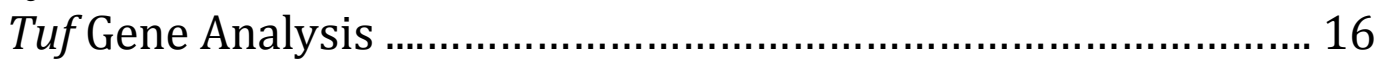

Flow Cytometric Detection and Quantification of Fecal Bacteria.17

Fluorescent Microscopy ............................................................. 18

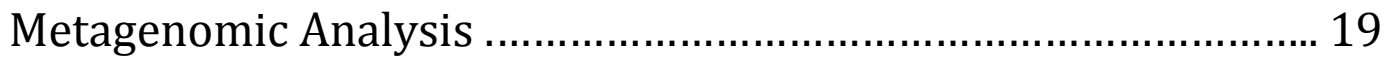

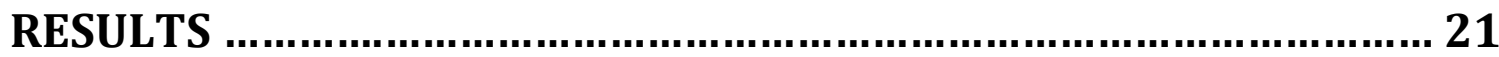

Gram stains of suspensions from fecal pellets from control mice and antibiotic-treated mice ........................................................ 21 Quantitative flow cytometry performed on fecal pellets from control and antibiotic-treated mice ............................................. 21

Fluorescent microscopy performed on fecal suspension to determine cellular composition 
Sequencing of V3 region of $16 \mathrm{~S}$ rRNA from DNA extracted from antibiotic-treated and control mice feces

Sequencing of V4 region of 16S rRNA from DNA extracted from antibiotic-treated and control mice feces ................................... 23

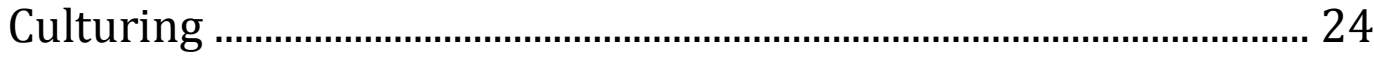

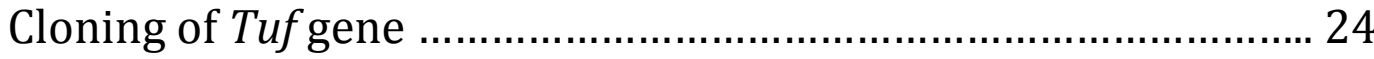

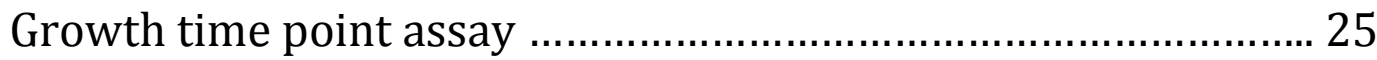

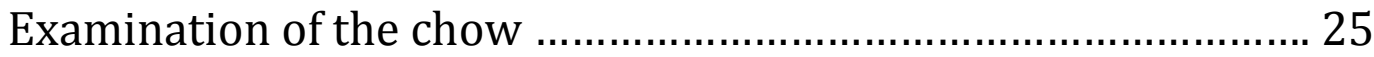

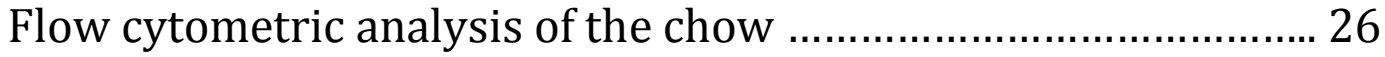

Live/Dead analysis .................................................................... 27

Effects of 2018 Teklad Global 18\% Protein Rodent Diet chow .... 27

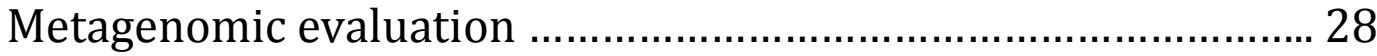

Detection of yeast in feces is dependent on chow ............................ 28

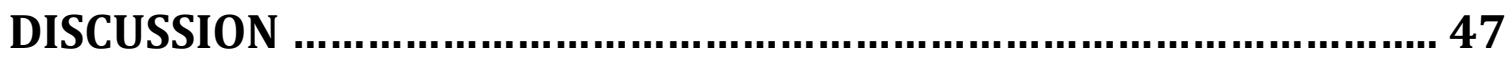

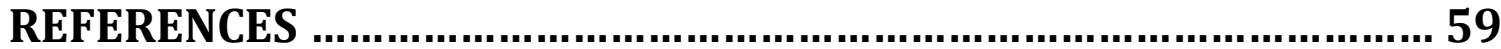




\section{LIST OF TABLES}

Table 1. Yeast in feces of antibiotic-treated mice dependent on

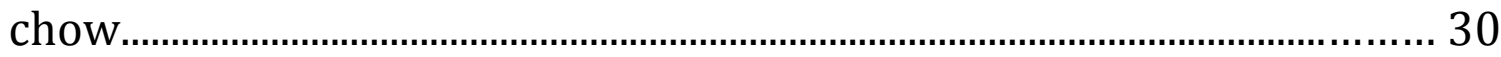

Table 2. Sequences of primers used in the construction of libraries for Illumina sequencing ............................................................................ 31

Table 3. Genus specific primers used to identify Lactococcus and Enterococcus. 32 


\section{LIST OF FIGURES}

Figure 1. Gram stains from fecal pellets from control mice and antibiotictreated mice.

Figure 2. Quantitative flow cytometry performed on suspension from fecal pellets from control and antibiotic-treated mice

Figure 3. Flourescent microscopy of fecal suspensions stained for bacterial identification 35

Figure 4. Taxonomical assignment of phylotypes found in feces from control and antibiotic-treated mice at the class level from V3 region of 16S rRNA sequencing analysis

Figure 5. Taxonomical assignment of phylotypes found in feces from control and antibiotic-treated mice at the class level from V4 region of $16 \mathrm{~S}$ rRNA sequencing analysis

Figure 6. Cultures of feces from antibiotic-treated mice 38

Figure 7. Culturing mouse fecal pellets and growth time point assay ....... 39

Figure 8. Gram stain analysis of Open Source and 2018 Teklad Global 18\% Rodent Diet chow 40

Figure 9. PCR analysis demonstrates Lactococcus DNA present in the Open Source Diet chow 41

Figure 10. Flow cytometric analysis of chow …………........................................ 42

Figure 11. Viability analysis of chow via flow cytometry .................................... 43

Figure 12. Statistical representation of the GI microbiome after sequencing V3 region of $16 \mathrm{~S}$ rRNA sequencing from antibiotic-treated and control mice fed Teklad Global chow

Figure 13. Metagenomic analysis 45

Figure 14. Quantitative flow cytometry performed on suspension from fecal pellets from control and antibiotic-treated mice on Teklad global chow......... 46 



\section{GENERAL INTRODUCTION}

From birth until death, microorganisms, especially bacteria, coexist with humans in a mutualistic relationship. The host provides nourishment and a suitable environment for the bacteria, and the bacteria play a role in establishing and maintaining a healthy immune system ${ }^{[1]}$, prevent colonization of pathogens ${ }^{[2]}$ and contribute to host nutrition by synthesizing vitamins (i.e. vitamin $\mathrm{K}$ and biotin) and metabolizing nondigestible starches ${ }^{[3-5]}$. There are an estimated $10^{14}$ bacterial cells (at least an order of magnitude more than the total number of our own cells) ${ }^{[6-7]}$ that colonize the human body, with the majority residing in areas that include the skin surface, oral cavity, nasal cavity, genitourinary system and the gut ${ }^{\{8\}}$. The compilation of microorganisms and their collective genome within a niche is referred to as a microbiome ${ }^{[9-11]}$, which can consist of bacteria, viruses, fungi and Archaea[12-14].

The intestinal microbiome has been identified as a key player in several processes that include; metabolic, nutritional, physiologic and immunologic ${ }^{[15]}$. The gut is sterile until the moment of birth, at which 
point the intestinal microbiome becomes established. The microbiome expands and increases in diversity through adolescence and into adulthood, at which point the population and diversity begins to decline ${ }^{[16]}$.

At birth, the intestinal microbiome can be influenced by means of delivery. A study found that babies delivered by vaginal birth had a significantly higher population of Actinobacteria than children delivered by caesarean section ${ }^{[17]}$. However, the microbiome quickly changes with the introduction of nutrients.

Intestinal microbiomes of newborns are greatly influenced by the source of nourishment they receive. Infants that are breast-fed have a larger population of Actinobacteria when compared to formula-fed infants, and have nearly a two-fold increase in total number of bacteria ${ }^{[18]}$. With the introduction of solid foods the microbiome takes a dramatic shift, and Actinobacteria percentages drop significantly while Bacteroidetes and Firmicutes become the dominant phyla ${ }^{[19] .}$ 
The trend of a microbiome that is primarily dominated by an equal ratio of Bacteroidetes and Firmicutes continues through adolescence and into adulthood. In adulthood, Firmicutes become the primary phylum in average, healthy adults microbiome. This trend continues throughout life as the number of bacteria and diversity decline ${ }^{[20] .}$

The human gut normally possesses one of the densest populations of bacteria found on Earth[21-22]. Populations of bacteria within the gut have been enumerated at levels reaching $10^{12}$ cells per ml of intestinal content with over 10,000 different phylotypes identified ${ }^{[22] . ~ M o l e c u l a r ~}$ approaches with Next-gen sequencing have detected that approximately only $1 \%$ of bacteria residing in the gut can be recovered by culturebased approaches ${ }^{[23]}$.

A variety of factors play a role in establishing and maintaining the gut microbiome to include: age, diet, health, geographic location and genetics $^{[24-30]}$. Dysbiosis within microbiomes have been linked to certain diseases: cancer, obesity, diabetes, atherosclerosis and inflammatory bowel disease [31-32]. 
Antibiotics introduced into the intestinal tract disrupt the microbiome by suppressing the total bacterial population and diversity in a variety of ways. Broad-spectrum antibiotics are often prescribed to treat infections to ensure eradication of the pathogenic bacteria. In the past little regard was taken to the effects that these antibiotics have on the normal microbiota, and how they could promote colonization of pathogens, such as Clostridium difficile. One study has shown that antibiotics introduced to infants are capable of modulating the microbiome even 8 wks after terminating antibiotic use ${ }^{[33] .}$

Antibiotic use became the standard of care for treating infections in the 1940s and has been widely used since. They have played a large role in extending human longevity; however antibiotic-resistant pathogens have wreaked havoc on health care and patients for at least the past two decades ${ }^{[34]}$. However, within the last decades with the improvements to high-throughput sequencing, the true significance of the microbiota within the gastrointestinal tract suppressing the colonizing of pathogens is being realized. 
Murine models of intestinal microbiome disruption are often used to demonstrate the effects of antibiotics. Antibiotics are typically administered either through drinking water or by oral gavages, and are used to selectively target groups of bacteria. Numerous cocktails of antibiotics have been used to either ablate the gut flora of mice to present a sterile gut environment or to target certain genera of bacteria $^{[35-41]}$. One recipe of antibiotics that is commonly used in studies is a combination of vancomycin, ampicillin, neomycin and metronidazole ${ }^{[36,39,42]}$. The metronidazole targets anaerobic bacteria, neomycin is effective against Gram-negative bacteria, vancomycin is highly effective against Gram-positive organisms, and the ampicillin targets the remaining bacteria and has broad spectrum capabilities. In these studies, it is evident that the antibiotics suppress either the selected commensal bacteria or ablate nearly the entire microbiome. An alternative approach to studying the microbial ecology of the small intestine is through the use of germ-free mice. One such study showed colonizing germ-free mice with known commensal bacteria was capable of preventing introduced pathogens from colonizing[43]. 
Prebiotics and probiotics have been studied for beneficial effects as supplements for the commensal bacteria in the gut. Prebiotics, such as cellulose and tagatose, are non-digestible food ingredients that modulate the gastrointestinal environment to promote the growth of select bacteria. A prebiotic mixture of galactooligosaccharides, brand name Bimuno, has demonstrated potential relief to patients suffering from inflammatory bowel disease. Probiotics are commensal bacteria, such as Lactobacillus and Bifidobacterium, which are commonly used to help augment existing commensal bacteria within the gut. There are conflicting reports on the clinical efficacy of probiotic use; however a ground-breaking study focused on fecal transplantations from healthy donors to treat patients with Clostridium difficile infections ${ }^{[44]}$. The results were remarkable, with greater than $95 \%$ of patients cleared of infection without a subsequent infection. Fecal transplants are currently being researched as potential treatments.

Apart from bacteria, yeast and Archaea play a role as commensal organisms within the human intestinal microbiome. It has been documented that yeast can propagate in response to suppressing bacterial growth through antibiotic-treatment. Interestingly, the 
lineage of the yeast changes under long-term antibiotic treatment ${ }^{[36]}$. Archaea, such as Methanobrevibacter smithii and Methanosphaera stadtmanae, are considered normal microbiota of the human intestines and have an essential role. ${ }^{[45]}$ As anaerobic bacteria metabolize pectin and other complex sugars, hydrogen and methanol are released as byproducts. Both hydrogen and methanol reduce the efficiency of fermentation, and these Archaea methanogens use both hydrogen and methanol as a source of energy. Although Archaea has not been identified to exist within the murine gut, given the similarities of composition and environment between the human and murine gut, it is reasonable to believe that Archaea could exist within the murine gut as a commensal organism.

The present study was undertaken to examine the role of diet in affecting the intestinal microbiome; particularly in the context of antibiotic-induced perturbations in an intestinal ecosystem. We initially wanted to add quantitative analysis to previously reported studies that relied on culture methods ${ }^{[46]}$ or $\mathrm{qPCR}^{[36]}$ to quantify perturbation of the16S gene in studies of the microbiome. We also wanted to explore the existence of Archaea within the murine intestinal microbiome. 


\section{MATERIALS AND METHODS}

\section{Mice}

Female C57BL/6 mice, aged 6 weeks old were obtained from The Jackson Laboratory (Bar Harbor, ME). The mice were randomly separated into two groups and housed in the Health Sciences Center Animal Care Facility 5 mice per cage under approved protocol number 12-0506.3. Upon arrival the mice were placed on either a controlled diet (referred to as Open Source chow), D12450B from Research Diets Inc (New Brunswick, New Jersey) or on 2018 Teklad Global 18\% Protein Rodent Diet chow ad libitum, dependent upon the experiment. The mice were allowed to acclimate for 5 days prior to use.

\section{Antibiotics}

The antibiotics vancomycin $(0.5 \mathrm{mg} / \mathrm{mL})$, ampicillin $(1.0 \mathrm{mg} / \mathrm{mL})$, metronidazole $(1.0 \mathrm{mg} / \mathrm{mL})$ and neomycin $(1.0 \mathrm{mg} / \mathrm{mL})$ were dissolved into filtered $(0.22 \mu \mathrm{m})$, sterile water and delivered to mice in lightprotected water bottles ${ }^{(35)}$. Aspartame $(3.75 \mathrm{mg} / \mathrm{mL})$ was also added to the antibiotic containing water to make the water palatable for the mice. 
All antibiotics were purchased through Sigma Aldrich (St Louis, MO). The vancomycin, ampicillin and neomycin were United States Pharmocopeia (USP) certified, and metronidazole was traceable to USP. The antibiotic water was made fresh every 48 hours, due to the loss of efficacy of the ampicillin in solution. Based on the minimum inhibitory concentration (MIC) against a laboratory strain of E. coli, it was determined that ampicillin lost $90 \%$ of its efficacy within 24 hours in solution. Due to the nature of the prolonged study and to recapitulate the results from Hildebrandt et al. [22], the decision to change the water at 48 hour intervals was made.

\section{Sample Collection and Processing}

To ensure that fresh fecal pellets were collected, mice were placed into sterile, empty pipette tip boxes for up to 3 minutes. The mice were under direct observation the entire time to ensure that once a fecal pellet was expelled, mice were placed back into their home cages and the pellets placed into $1.5 \mathrm{~mL}$ Eppendorf tubes. If the fecal pellets were contaminated by urine, the samples were discarded. The samples were weighed and then emulsified in $1 \mathrm{~mL}$ of sterile water using a Pellet Pestle Motor from Kimble Chase Kontes (Vineland, NJ). The samples 
were then centrifuged at $800 \mathrm{x} g$ for 1 minute to remove fecal debris. Five hundred uL of the supernatants were diluted in $500 \mathrm{uL}$ of sterile water in a fresh $1.5 \mathrm{~mL}$ Eppendorf tubes.

\section{DNA Isolation}

DNA was isolated from murine fecal pellets by processing fecal pellets as described above and purifying the DNA using QIAamp DNA Stool Mini Kit from Qiagen (Valencia, CA, USA) following the manufacturer's instructions. Genomic DNA was stored at $-20^{\circ} \mathrm{C}$.

\section{PCR and Fragment Purification}

The PCR primer sequences, the conditions for amplification of the V3 region of the $16 \mathrm{~S}$ ribosomal RNA gene, and multiplexed DNA sequencing strategy were as described in Bartram et al.[47] unless otherwise indicated. The PCR primers for the V4 region of the $16 \mathrm{~S}$ ribosomal RNA gene were as described in Caparoso et al..[48]. High-pressure liquid chromatography purified PCR primers for the V3 region were obtained from Integrated DNA Technologies (Coralville, IA, USA) and the V4 region primers were obtained from Invitrogen (Grand Island, NY, USA). Bacterial DNA was amplified using an AccuPrime PCR Kit (Invitrogen 
Life Technologies, Grand Island, NY, USA) on a Techne Genius Model FGEN02TP Thermal Cycler using the following conditions: $95^{\circ} \mathrm{C}$ for 6 minutes denature; $95^{\circ} \mathrm{C}$ for 2 minutes, $50^{\circ} \mathrm{C}$ for 2 minutes, $72^{\circ} \mathrm{C}$ for 2 minutes 30 cycles; $72^{\circ} \mathrm{C}$ for 4 minutes extend. Each reaction contained 1 U TAQ polymerase, $5 \mu \mathrm{l}$ 10x buffer 1(600 mM Tris-SO4 (pH 8.9), 180 mM (NH4)2SO4, 20 mM MgSO4, 2 mM dGTP, 2 mM dATP, 2 mM dTTP, 2 mM dCTP, thermostable AccuPrime ${ }^{\mathrm{TM}}$ protein, $10 \%$ glycerol), $20 \mu \mathrm{M}$ forward primer, $20 \mu \mathrm{M}$ reverse primer, and up to $60 \mathrm{ng}$ DNA in a total volume of $50 \mu \mathrm{l}$. PCR reactions were performed in triplicate and reaction products were pooled prior to purification. Because there was a low concentration of DNA in the samples from the treated mice, it was necessary to perform 30 cycles of amplification to obtain sufficient material to view on gels. Pooled PCR products were purified by electrophoresis through 2\% agarose in Tris/acetate/EDTA gels and the bands corresponding to approximately 300 base pairs for V3 and bands corresponding to approximately 400 base pairs for V4 were excised and purified using a QIAquick Gel Extraction Kit (Qiagen, Valencia,CA, USA) according to the manufacturer's directions. The V3 and V4 regions both vary in length by about 30 base pairs among different species of bacteria, and the sequences obtained and analyzed in this study showed 
similar size variability. The amplicon of the V3 region ranged from 296 to 327 base pairs, of which 160 base pairs were the primer. The amplicon of the $\mathrm{V} 4$ region ranged from 373 to 402 base pairs, of which 172 base pairs were the primer.

\section{PCR Amplification with Genus Specific Primers}

Bacterial DNA was amplified with either on a Techne Genius Model FGEN02TP Thermal Cycler using the following conditions: $95^{\circ} \mathrm{C}$ for 6 minutes denature; $95^{\circ} \mathrm{C}$ for 2 minutes, $50^{\circ} \mathrm{C}$ for 2 minutes, $72^{\circ} \mathrm{C}$ for 2 minutes 20 cycles; $72^{\circ} \mathrm{C}$ for 4 minutes extend. Each reaction contained 1 U TAQ polymerase, $5 \mu \mathrm{l}$ 10x buffer 1(600 mM Tris-SO4 (pH 8.9), 180 mM (NH4)2SO4, 20 mM MgSO4, 2 mM dGTP, 2 mM dATP, 2 mM dTTP, 2 $\mathrm{mM}$ dCTP, thermostable AccuPrime ${ }^{\mathrm{TM}}$ protein, $10 \%$ glycerol), $20 \mu \mathrm{M}$ forward primer, $20 \mu \mathrm{M}$ reverse primer, and up to $100 \mathrm{ng}$ DNA in a total volume of $50 \mu \mathrm{l}$. The Lactococcus ${ }^{[61]}$ and Enterococcus ${ }^{[62]}$ specific primers are listed in Table 3.

\section{Illumina HiSeq High Throughput (HT) Sequencing}

Libraries were sequenced in a $2 \times 125$ bp paired-end strategy on an 
Illumina HiSeq1000, so that the forward and reverse reads could be assembled into a single contig. Indexed libraries were pooled so that 12 libraries were sequenced in each lane of the flow cell. Eight pmols of the pooled libraries were clustered onto an Illumina v2 sequencing flow cell using an Illumina cBOT. Reads were converted from Illumina bcl format to fastq format and separated into bins based on exact match to the index using CASAVA 1.8.2 (Illumina, San Diego CA, USA). The Illumina HiSeq was performed at Marshall University (Huntington, West Virginia.) Sanger sequencing was performed by the Molecular Medicine core facility at West Virginia University and MiSeq sequencing was performed by the West Virginia University Genomics core facility.

\section{HiSeq Data Processing}

Sequence files were initially processed by removing sequences corresponding to linkers and primers by automated batch processing using scripts written in-house. In an effort to reduce artifacts generated by sequencing errors, a strict quality filtering protocol was employed that reduced the number of analyzed sequences to approximately $35 \%$ of the total number of sequences generated. Nevertheless, an average of more than two million high quality reads was obtained from each 
sample. Quality filtering of DNA sequences was performed using the following steps: 1) Sequences were first filtered by the Illumina software to eliminate the poorest reads $(Q$ score $\geq 30)$ and imperfect primer matches. 2) The forward and reverse sequences were matched to construct a sequence that spanned the entire region between the primers with a program written in-house. The original Illumina sequences were all 125 bases in length, which is where the run ended. The pairing strategy overlaid the two 3' ends starting with an overlap of 58 bases for the V3 region. The overlap window was extended one base at a time to 89 bases until a perfect match was obtained in the overlap region. Any pair of sequences that did not match at $100 \%$ identity in any of the size windows was discarded. This step eliminated $56 \%$ of the sequences, which overwhelmingly had the lowest quality scores. In general, the sequence quality was better in the middle than at the end, so this preferentially eliminated sequences with sequencing artifacts. 3) Paired sequences with a Phred quality score of less than five were discarded. This removed a few remaining low quality sequences, especially any that had low quality in the regions between the primers and the overlap. 4) The sequences were clustered by matching against the Greengenes database, which is a curated collection of known 
bacterial 16S sequences. Sequences that did not match any of the known bacterial sequences with $97 \%$ identity were discarded. This removed chimeras and most major PCR artifacts and represented approximately $5 \%$ of the total remaining sequences. Matching at $97 \%$ identity meant that any single base PCR artifacts would be combined with the corresponding authentic sequence (since the region is about 100 bases long, up to three single base changes will be ignored). 5) The resulting table of operational taxonomic units (OTUs) was filtered to remove any sequences that appeared less than 150 times. Because of the length of the V4 region, we were unable to perform the paired end strategy. Scripts written in-house in biopython were used to convert the filtered Illumina data to the FASTA format for analysis by QIIME for taxonomic assignment and measurements of microbial diversity, but scripts to do this are now part of QIIME. To process Illumina generated files in QIIME, the file headers were changed to begin with '>sample_number' where ' sample' is the sample number and 'number' is the number of the sequence in the file. All of the sequences were then combined into one file for analysis with QIIME. 


\section{QIIME}

All QIIME analyses were performed on a virtual server hosted by Amazon Web Services using an existing QIIME image. The server had the following specification: QIIME 1.8.0 EBS East XLARGE (ami438d5b2a). The following QIIME scripts were used during analysis and default parameters were used unless otherwise noted: 1) ' pick_ reference_otus_through_otu_table.py' matched sequences at 97\% sequence identity with OTUs associated with specific bacterial phylotypes in the Greengenes database (4Feb2014); 2) 'summarize_taxa_through_plots.py' generated bar graphs of the relative abundance of different taxa in each sample; 3) ' alpha_rarefaction.py' generated alpha rarefication plots; 4) 'pick_rep_set.py', ' align_seqs.py' ,

'filter_alignment.py', and ' make_phylogeny.py' were chained to generate a phylogenetic tree of the OTUs.

\section{Tuf Gene Analysis}

The tufgene encoding elongation factor Tu was amplified with the tuf forward primer: (5'-CCAATGCCACAAACTCGT-3') and the tuf reverse primer (5'-CCTGAACCAACAGTACGT-3') as described in Li et al. [49]. 
Cloning of the tuf gene was carried out using a TOPO® TA for sequencing kit from Invitrogen (Grand Island, NY, USA) according to the manufacturer's instructions. The 830 bp tuf gene was amplified by PCR using AccuPrime PCR Kit (Invitrogen Life Technologies, Grand Island, NY, USA) on a Techne Genius Model FGEN02TP Thermal Cycler using the following conditions: $95^{\circ} \mathrm{C}$ for 6 minutes denature; $95^{\circ} \mathrm{C}$ for 2 minutes, $50^{\circ} \mathrm{C}$ for 2 minutes, $72^{\circ} \mathrm{C}$ for 2 minutes 30 cycles; $72^{\circ} \mathrm{C}$ for 4 minutes extend. Each reaction contained $0.5 \mu \mathrm{l}$ TAQ polymerase, $5 \mu \mathrm{l}$ 10x buffer 1(600 mM Tris-SO4 (pH 8.9), 180 mM (NH4)2SO4, $20 \mathrm{mM}$ MgSO4, 2 mM dGTP, 2 mM dATP, 2 mM dTTP, 2 mM dCTP, thermostable AccuPrime ${ }^{\mathrm{TM}}$ protein, $10 \%$ glycerol), $20 \mu \mathrm{M}$ forward primer, $20 \mu \mathrm{M}$ reverse primer, and up to $60 \mathrm{ng}$ DNA in a total volume of $50 \mu \mathrm{l}$. For cloning, the amplified fragment was inserted into the pCR4-TOPO plasmid (4.0 Kb). Electrically competent cells (E. coli TOP10) were transformed with the insert-containing plasmids. The recombinant clones were selected by growth on LB agar with ampicillin $(100 \mu \mathrm{g} / \mathrm{ml})$ following incubation for $18 \mathrm{hrs}$ at $37^{\circ} \mathrm{C}$ with $5 \% \mathrm{CO} 2$. The presence of the insert in E. coli TOP10 transformants was verified by colony PCR using the tuf primers. Plasmids from transformants were extracted using the Mini-Prep 96 from Macconnell Research [San Diego, CA, USA] 
according to the manufacturer's instructions. Five mL cultures grown in Magnificent Broth with ampicillin were incubated overnight. $2 \mathrm{~mL}$ of the cultures were placed in each well and applicator combs inserted. Lysis tablets were then added and allowed to incubate at room temp for 15 mins. Purified DNA was then removed from the corresponding wells on the back of the cartridge and the samples were sequenced by WVU Genomics Core facility by capillary (Sanger) sequencing.

\section{Flow Cytometric Detection and Quantification of Fecal Bacteria}

Fifty uL of the suspensions from fecal pellets were diluted in $950 \mathrm{uL}$ of sterile $0.5 \% \mathrm{NaCl}$ for a final dilution of $1 / 400$. Utilizing Invitrogen's (Grand Island, NY, USA) Bacteria Counting Kit for flow cytometry, $1 \mu \mathrm{L}$ of SYTO BC stain was added to the diluent as well as $10 \mu \mathrm{L}$ of $1 \times 10^{8}$ /mL of microspheres, per manufacturer's instructions. Samples were analyzed with a Becton Dickinson FACSCalibur using the following settings: FSC E00 gain; SSC 350 voltage; FL1 412 voltage; FL2 550 voltage and FL3 650 voltage. A region was placed around the beads to capture a quantity of 10,000 beads; another region was placed to capture the number of bacteria that were counted in comparison to the 
10,000 beads. WINMDI 2.8 software, (Joe Trotter, Scripps Research Institute, La Jolla, CA) was used to analyze the flow cytometer data.

\section{Fluorescent Microscopy}

Fifty $\mu \mathrm{L}$ of the suspensions from fecal pellets were diluted in $950 \mu \mathrm{L}$ sterile $0.85 \% \mathrm{NaCl}$, either $1 \mu \mathrm{L}$ of BacLight Red bacterial stain (Invitrogen, Grand Island, NY, USA), $1 \mu \mathrm{L}$ of SYTO 9 stain (Invitrogen, Grand Island, NY, USA) , or $1 \mu \mathrm{L}$ of both were added and samples incubated for 15 minutes at room temperature in the dark. Ten $\mu \mathrm{L}$ of sample was then placed on glass microscope slides, 1 drop of ProLong Gold (Invitrogen, Grand Island, NY, USA) was added and a cover slip applied. The slides incubated for 24 hours at room temperature in the dark to allow the coverslip to seal in accordance with manufacturer's instructions, and were examined by confocal microscopy.

\section{Metagenomics analysis}

Predictive functional profiling of the microbiome was performed using the $16 \mathrm{~S}$ rRNA sequencing data and then running the following scripts through the PICRUSt database:[50] 1) normalize_by_copy_number.py; 2) 
predict_metagenomes.py; 3) categorize_by_function.py. QIIME commands were then executed on the file: 1) summarize_taxa.py (with md_identifier=KEGG_Pathways flag); 2) plot_taxa_summary.py. 


\section{RESULTS}

\section{Gram stains of suspensions from fecal pellets from control mice}

\section{and antibiotic-treated mice}

Suspensions of fecal pellets from both control and antibiotic-treated mice were collected after two weeks of treatment and analyzed by Gram stain. Samples from control mice displayed a very diverse population of bacteria, which consisted of both Gram-positive and Gram-negative rods and cocci and was consistent with normal gastrointestinal microbiota (Fig 1A). Gram stains of samples from the antibiotic-treated mice showed distinctly homogeneous Gram-positive coccobaccilli, as well as some yeast (Fig 1B), suggesting that antibiotic treatment altered the normal intestinal microbiota.

\section{Quantitative flow cytometry performed on fecal pellets from}

\section{control and antibiotic-treated mice}

Quantitative cell counts were performed on suspensions of fecal bacteria by flow cytometry. Ten samples from each group were analyzed and an approximate $1-\log _{10}$ reduction in the number of bacteria was found in the antibiotic-treated samples when compared to 
the control samples (Fig 2A). As was observed on the Gram stains, the control mice had a diverse population (Fig 2B) of bacteria as determined by forward scatter, while the antibiotic-treated mice had a homogeneous population (Fig 2C).

\section{Fluorescent microscopy performed on fecal suspension to determine cellular composition}

To confirm that the Gram stain and flow cytometric assays were detecting bacteria, samples were stained with dual fluorescent stains (Fig 3A and B) and examined using confocal microscopy. A merged view (Fig 3C) displays that the putative bacteria contain nucleic acid and their cell walls are comprised of peptidoglycan.

\section{Sequencing of V3 region of 16S rRNA from DNA extracted from antibiotic-treated and control mice feces}

Paired-end sequencing of the V3 region of the 16S rRNA gene was performed to identify the Gram-positive coccobaccilli in the antibiotictreated mice, and characterize the fecal microbiomes of the treated and non-treated mice. Figure 4 represents a graphic display of the Organizational Taxonomic Units (OTU) table generated from QIIME, and 
shows substantial increase in Lactococcus species in the antibiotictreated group when compared to the control group. Within the antibiotic-treated group, there is a decrease in Bacteroidetes and nonLactococcus, Firmicutes. Although the Lactococcus is present in the control group as well, it is in much smaller quantities when compared to the antibiotic-treated samples.

\section{Sequencing of V4 region of 16S rRNA from DNA extracted from}

\section{antibiotic-treated and control mice feces}

Due to the length of the V4 region of the 16S rRNA gene, contigs could not be generated as were with the $\mathrm{V} 3$ region because of only 1 or 2 base overlaps. The V4 region sequencing (Fig 5) displayed a loss of Bacteroidetes and an increase in Lactococcus in the antibiotic-treated samples when compared to the control samples, albeit the changes were not as dramatic in the V4 results as displayed in the V3 results. A small percentage of Archaea was also detected in antibiotic-treated samples.

\section{Culturing}

Numerous attempts at culturing the coccobacilli that were observed by Gram stain in the antibiotic-treated samples yielded predominantly 
overgrowth of yeast (Fig 6A). Upon closer examination, there were also scant pinpoint, $\alpha$-hemolytic colonies. These colonies were isolated (Fig 6B), DNA was extracted, and the entire 16S rRNA gene was PCRamplified and sequenced. Sequence analysis identified the alpha hemolytic colonies as Enterococcus casseliflavus, not Lactococcus. The inconsistency between the HT sequencing and culture results required additional analysis.

\section{Cloning of Tuf gene}

Sequence variation in the tuf gene, a prokaryotic elongation factor, has been previously used to differentiate species of Gram-positive cocci ( $\mathrm{Li}$ et al.[39] Therefore fecal DNA from antibiotic-treated mice was amplified using degenerate tuf-specific primers, cloned, and sequenced.

14 of 14 samples yielded a 99\% match with Lactococcus through BLAST. These results confirmed the results from the V3 sequencing data.

\section{Growth time point assay}

After numerous unsuccessful attempts to grow Lactococcus from the fecal samples from antibiotic-treated mice on a variety of bacterial media, fecal samples from the antibiotic-treated mice were cultured in 
evaporated milk. Aliquots were removed at time 0 , and every 8 hours of incubation for a 24-hour period. DNA samples from each time point were amplified using Lactococcus or Enterococcus specific primers. The reactions resulted in an initial positive reaction for the Lactococcus with no increase in the levels of PCR product through the 24-hour time period, indicating that the Lactococcus was not replicating. However, although the initial sample failed to amplify with Enterococcus specific primers, weak positive results were detected at 16 hours and strong positive observed at 24-hours (Fig 7A). Gram stains were performed on the sample at 24 hours and demonstrated Gram-positive coccobaccili detected and yeast in the culture (Fig 7B).

\section{Examination of the chow}

To identify the source of the Lactococcus, a $20 \mathrm{mg}$ sample of the Open Source Chow was processed in the same manner as the fecal samples and Gram stained (Fig 8A). The Gram stain results of the Open Source chow demonstrated that the chow was heavily contaminated with Gram-positive coccobaccili, with no yeast observed. A sample of the 2018 Teklad Global 18\% Protein Rodent Diet chow normally used in HSC vivarium was then examined in the same manner as the Open 
Source chow and Gram stained (Fig 8B). No bacteria were visualized on the Gram stain of the 2018 Teklad Global 18\% Protein Rodent Diet chow. To determine if the bacteria from the Open Source chow was Lactococcus, PCR reactions were performed on both the Open Source chow and the 2018 Teklad Global 18\% Protein Rodent Diet chow with both specific and universal primers for 16S rRNA (Fig 9). The results demonstrated that both the 2018 Teklad Global 18\% Protein Rodent Diet chow and the Open Source chow contained DNA from bacteria, however the Open Source Chow contained Lactococcus DNA.

\section{Flow cytometric analysis of the chow}

The Open Source and 2018 Teklad Global 18\% Protein Rodent Diet chows were analyzed by flow cytometry to compare the forward scatter characteristics of bacteria in the chow with bacteria in the fecal samples of antibiotic-treated mice. The Open Source chow had a distinct bacterial population that resembled the fecal samples from the antibiotic-treated mice (Fig 10A), while the 2018 Teklad Global 18\% Protein Rodent Diet chow lacked a distinct population (Fig 10B). A pure culture of Lactococcus was analyzed for comparison (Fig 10C). 
Quantitative analysis revealed the bacterial cell count for the Open Source chow to be $3 \times 10^{12}$ per gram.

\section{Live/Dead Analysis}

To determine the viability of the Lactococcus present within the Open Source chow, a Live/Dead analysis was performed using Invitrogen's LIVE/DEAD BacLight Bacterial Viability kit according to manufacturer's instructions. The analysis showed that the Lactococcus from the Open Source chow was non-viable (Fig 11).

\section{Effects of 2018 Teklad Global 18\% Protein Rodent Diet chow}

To determine the differences of gastrointestinal microbiomes when the mice were placed on different chow, 15 additional C57BL/6 mice were acquired from Jackson Laboratory. Upon receipt, the mice were handled in the exact manner as before with the exception of placing the mice on 2018 Teklad Global 18\% Protein Rodent Diet chow rather than the Open Source Diet. DNA extracted from fecal pellets from both antibiotic-treated and control mice were amplified using V3 16S rRNA primers and sequenced (Fig 12) using an Illumina MiSeq. The antibiotic-treated mice produced an overwhelming dominance- >95\% 
in all 5 mice- of a lactose fermenting Enterobacteriaceae. The short V3 regions of the $\mathrm{V} 3$ region of the $16 \mathrm{~S}$ rRNA were unable to differentiate between species of Enterobacteriaceae due to homology of the genus within the V3 region. With the removal of the Open Source Diet no Lactococcus was detected.

\section{Metagenomic evaluation}

PICRUSt Bioinformatics software was utilized to predict metagenomic functions from the $16 \mathrm{~S}$ rRNA sequencing data (Fig 13.) The most notable change was a reduction in the content of genes predicted to play a role in motility, which correlates with Lactococcus being a non-motile organism.

\section{Detection of yeast in feces is dependent on chow}

Ingestion of the 2018 Teklad Global 18\% Protein Rodent Diet chow not only produced a dominant Enterobacteriaceae, but also failed to produce yeast in the feces of antibiotic-treated mice. Yeast was not detected in the feces of the mice that were fed 2018 Teklad Global 18\% Protein Rodent Diet chow and antibiotic treatment for 10 weeks by either culture or Gram stain. Another group of mice that were on 
antibiotic treatment and fed the 2018 Teklad Global 18\% Protein

Rodent Diet chow for 3 weeks and then converted to Open Source chow for the remaining 7 weeks also produced the Enterobacteriaceae with the absence of yeast. In comparison, the mice that were on antibiotic treatment and the Open Source chow displayed yeast by culture and Gram stain after 2 weeks. (Data not shown) 


\begin{tabular}{|l|c|}
\hline \multicolumn{1}{|c|}{ Table 1. Yeast in feces of antibiotic-treated mice dependent on chow } \\
\hline \multicolumn{1}{|c|}{ Chow } & Yeast \\
\hline Open Source & + \\
\hline Teklad Global & - \\
\hline Teklad Global/converted to Open Source* & - \\
\hline
\end{tabular}

*Mice were fed Teklad Global chow for 3 wks and then converted to Open Source chow for remaining 7 weeks 
Table 2. Sequences of primers used in the construction of libraries for Illumina sequencing. Lowercase letters denote adapter sequences necessary for binding to the flow cell, underlined lowercase are binding sites for the Illumina sequencing primers, bold uppercase highlight the index sequences and regular uppercase are the $\mathrm{V} 3$ or $\mathrm{V} 4$ region primers. ${ }^{[37]}$

\begin{tabular}{|c|c|}
\hline \multicolumn{2}{|c|}{ Forward Primers Sequence (5' to 3') } \\
\hline V3_Fmod & aatgatacggcgaccaccgagatctacactctttccctacacgacgctcttccgatctNNNNCCTACGGGAGGCAGCAG \\
\hline V4_Fmod & aatgatacggcgaccaccgagatctacactctttccctacacgacgctcttccgatctNNNNGTGCCAGCGCCGCGGTAA \\
\hline \multicolumn{2}{|c|}{ Reverse Primers } \\
\hline V3_1R & caagcagaagacggcatacgagatCGTGATgtgactggagttcagacgtgtgctcttccgatctATTACCGCGGCTGCTGG \\
\hline V3_2R & caagcagaagacggcatacgagatACATCGgtgactggagttcagacgtgtgctcttccgatctATTACCGCGGCTGCTGG \\
\hline V3_3R & caagcagaagacggcatacgagatGCCTAAgtgactggagttcagacgtgtgctcttccgatctATTACCGCGGCTGCTGG \\
\hline V3_4R & 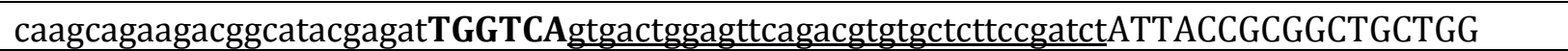 \\
\hline V3_5R & caagcagaagacggcatacgagatCACTGTgtgactggagttcagacgtgtgctcttccgatctATTACCGCGGCTGCTGG \\
\hline V3_6R & caagcagaagacggcatacgagatATTGGCgtgactggagttcagacgtgtgctcttccgatctATTACCGCGGCTGCTGG \\
\hline V3_7R & caagcagaagacggcatacgagatGATCTGgtgactggagttcagacgtgtgctcttccgatctATTACCGCGGCTGCTGG \\
\hline V3_8R & caagcagaagacggcatacgagatTCAAGTgtgactggagttcagacgtgtgctcttccgatctATTACCGCGGCTGCTGG \\
\hline V3_9R & caagcagaagacggcatacgagatCTGATCgtgactggagttcagacgtgtgctcttccgatctATTACCGCGGCTGCTGG \\
\hline V3_10R & 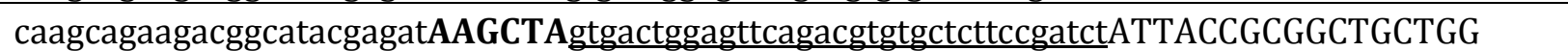 \\
\hline V3_11R & $\begin{array}{l}\text { caagcagaagacggcatacgagatGTAGCCgtgactggagttcagacgtgtgctcttccgatctATTACCGCGGCTGCTGG } \\
\end{array}$ \\
\hline V3_12R & caagcagaagacggcatacgagatTACAAGgtgactggagttcagacgtgtgctcttccgatctATTACCGCGGCTGCTGG \\
\hline V4_13R & caagcagaagacggcatacgagatCGTACT gtgactggagttcagacgtgtgctcttccgatctGGACTACHVGGGTWTCTAAT \\
\hline V4_14R & caagcagaagacggcatacgagatGACTGAgtgactggagttcagacgtgtgctcttccgatctGGACTACHVGGGTWTCTAAT \\
\hline V4_15R & caagcagaagacggcatacgagatGCTCAAgtgactggagttcagacgtgtgctcttccgatctGGACTACHVGGGTWTCTAAT \\
\hline V4_16R & caagcagaagacggcatacgagatTCGCTTgtgactggagttcagacgtgtgctcttccgatctGGACTACHVGGGTWTCTAAT \\
\hline V4_17R & caagcagaagacggcatacgagatTGAGGAgtgactggagttcagacgtgtgctcttccgatctGGACTACHVGGGTWTCTAAT \\
\hline V4__18R & caagcagaagacggcatacgagatACAACCgtgactggagttcagacgtgtgctcttccgatctGGACTACHVGGGTWTCTAAT \\
\hline V4__19R & caagcagaagacggcatacgagatACCTCAgtgactggagttcagacgtgtgctcttccgatctGGACTACHVGGGTWTCTAAT \\
\hline V4__20R & caagcagaagacggcatacgagatACGGTAgtgactggagttcagacgtgtgctcttccgatctGGACTACHVGGGTWTCTAAT \\
\hline V4_21R & caagcagaagacggcatacgagatAGTTGGgtgactggagttcagacgtgtgctcttccgatctGGACTACHVGGGTWTCTAAT \\
\hline V4_22R & caagcagaagacggcatacgagatCTCTCTgtgactggagttcagacgtgtgctcttccgatctGGACTACHVGGGTWTCTAAT \\
\hline V4_23R & caagcagaagacggcatacgagatCAAGTGgtgactggagttcagacgtgtgctcttccgatctGGACTACHVGGGTWTCTAAT \\
\hline V4_24R & caagcagaagacggcatacgagatCCTTGAgtgactggagttcagacgtgtgctcttccgatctGGACTACHVGGGTWTCTAAT \\
\hline
\end{tabular}


Table 3. Genus specific primers used to identify Lactococcus and Enterococcus.

\begin{tabular}{|l|l|}
\hline \multicolumn{2}{|l|}{ Lactococcus specific primers (5' to $3^{\prime}$ ) } \\
\hline LacF & GTACTTGTACCGACTGGAT \\
\hline LacreR & GGGATCATCTTTGAGTGAT \\
\hline & \\
\hline Enterococcus specific primers (5' to $3^{\prime}$ ) \\
\hline Ent1 & TACTGACAAACCATTCATGATG \\
\hline Ent2 & AACTTCGTCACCAACGCGAAC \\
\hline
\end{tabular}




\section{A}

B
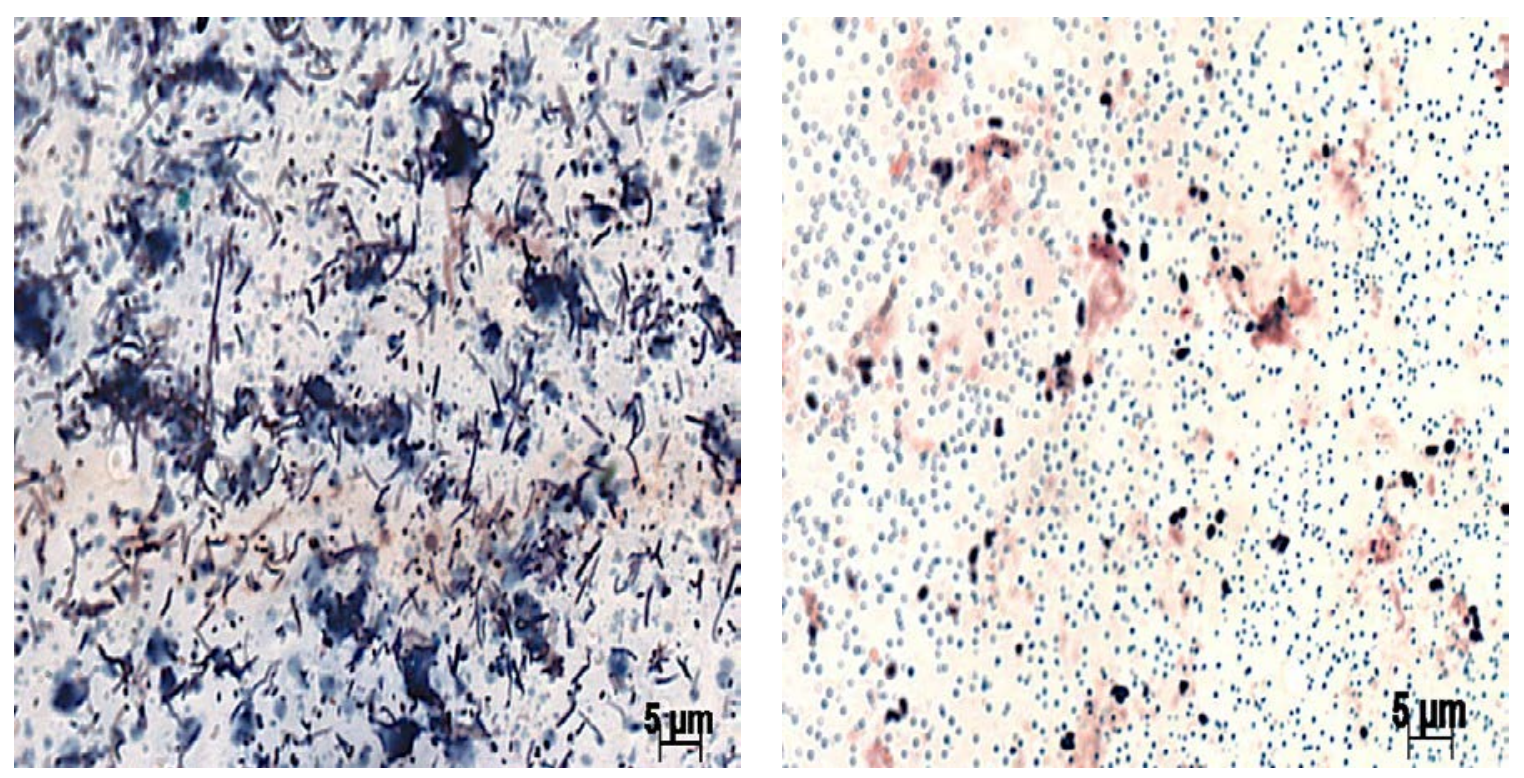

Figure 1. Gram stains from fecal pellets from control mice (A) and

antibiotic-treated mice (B). In (A) there is a very diverse population of both Gram-positive and Gram-negative organisms both rods and cocci.

Samples from antibiotic-treated mice (B) showed distinct homogeneous

Gram-positive coccobaccilli, as well as yeast. Photos are taken at 630X. 
A

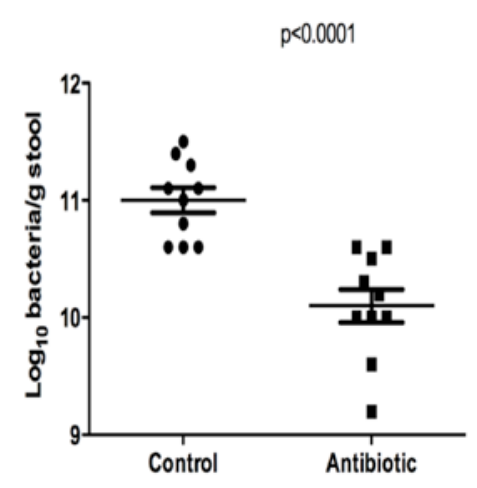

B

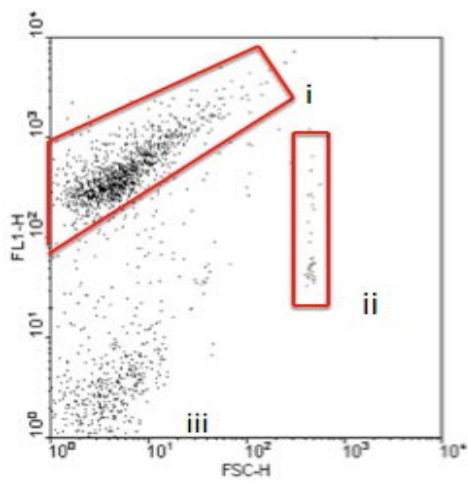

C

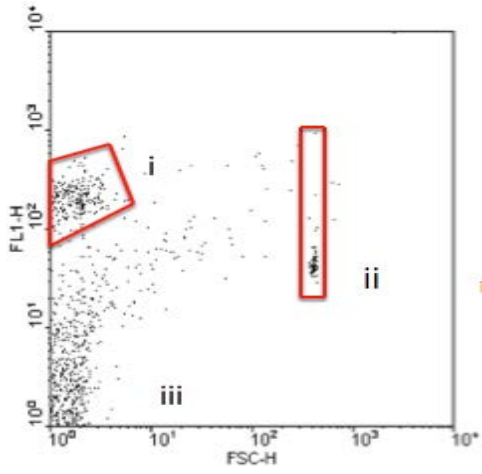

Figure 2. Quantitative flow cytometry performed on suspension from fecal pellets from control and antibiotic-treated mice on Open Source diet. Samples from 10 mice in each group were analyzed and displayed an average of a $1-\log _{10}$ difference in bacterial load (A). A heterogeneous population of cells is displayed in the control sample (B), when compared to the antibiotic-treated sample (C), which displays a distinct homogenous population of bacteria. (i) bacterial cells population, (ii) microspheres population, (iii) debris. 
A

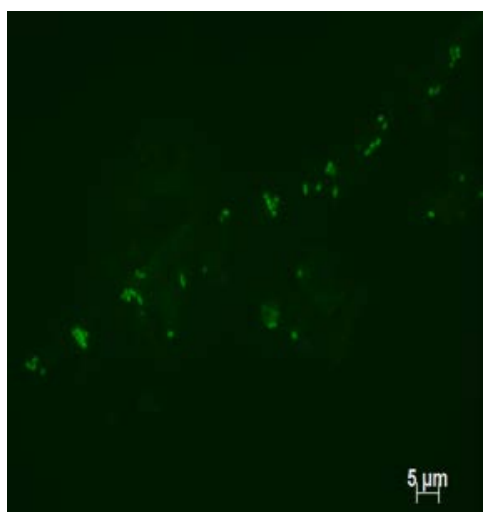

B

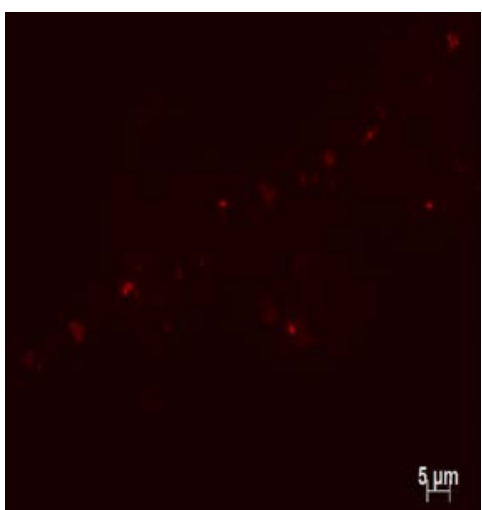

C

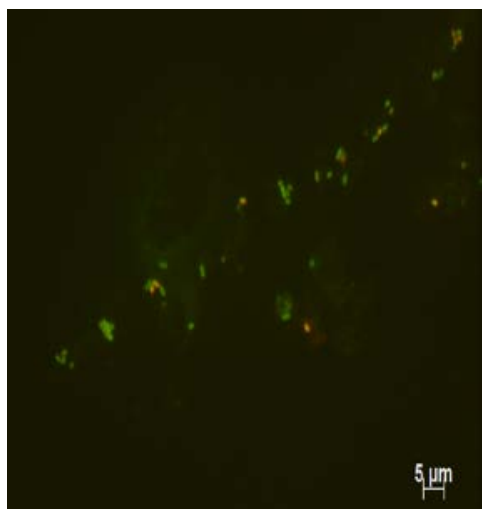

Figure 3. Fluorescent microscopy of fecal suspensions stained for bacterial identification. Utilizing a dual stain; Syto 9, which stains the nucleic acid green (A), a peptidoglycan stain that fluoresces red (B) and then merging $(C)$, confirmed that the cells contained DNA and peptidoglycan. Photos are taken at 630X. 


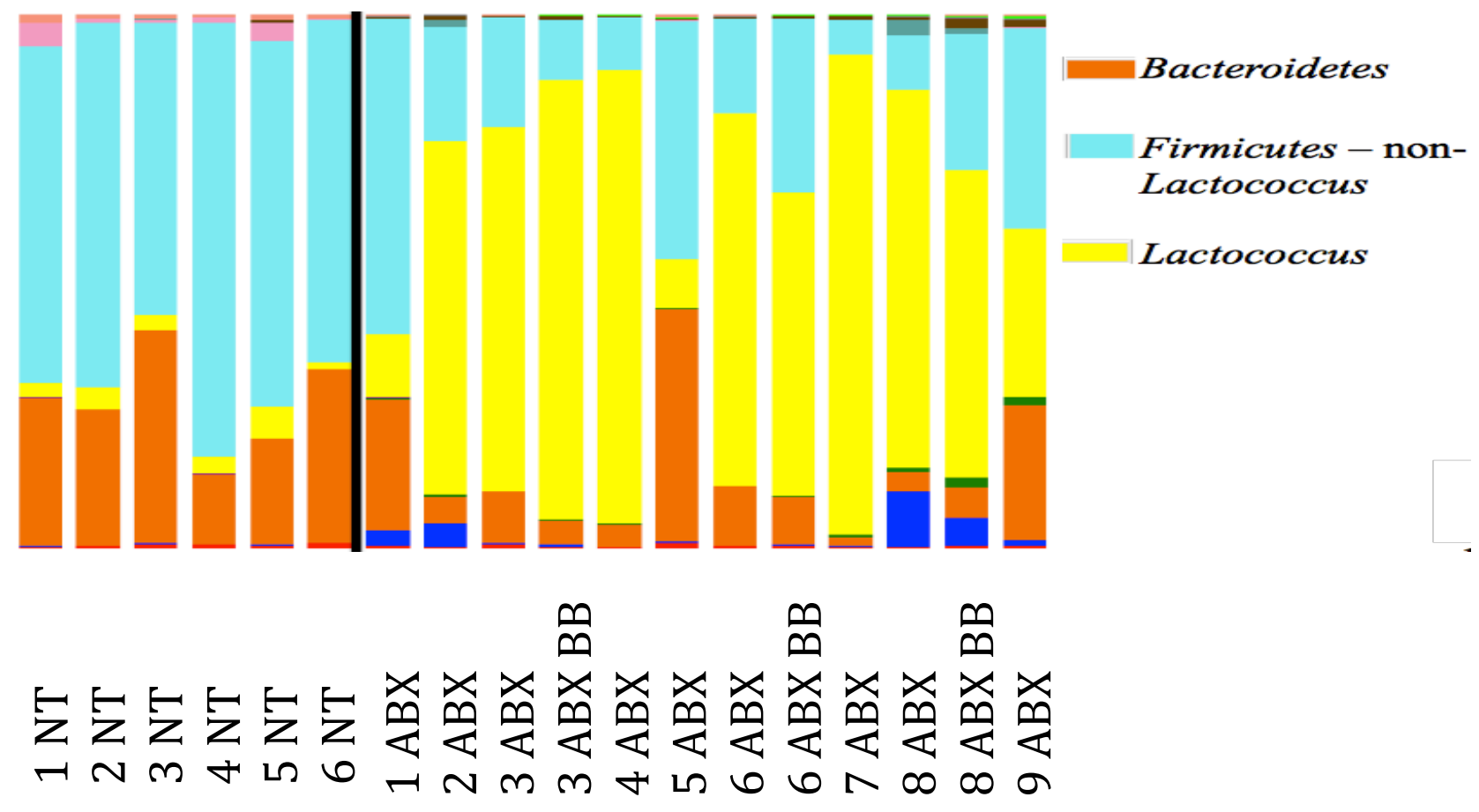

Figure 4. Taxonomic assignment of phylotypes found in feces from control and antibiotic-treated mice at the class level from $\mathrm{V} 3$ region 16S rRNA sequencing analysis. The total height of the bar represents $100 \%$ of the assigned sequences after quality filtering, and the height of the individually colored bars represents the percentage of the class of organisms. The expansion of Lactococcus within the antibiotic-treated group is shown, while they also lose a large percentage of other Firmicutes and Bacteroidetes. The control mice had a small percentage of Lactococcus, but displayed a larger heterogeneity of bacterial population. 


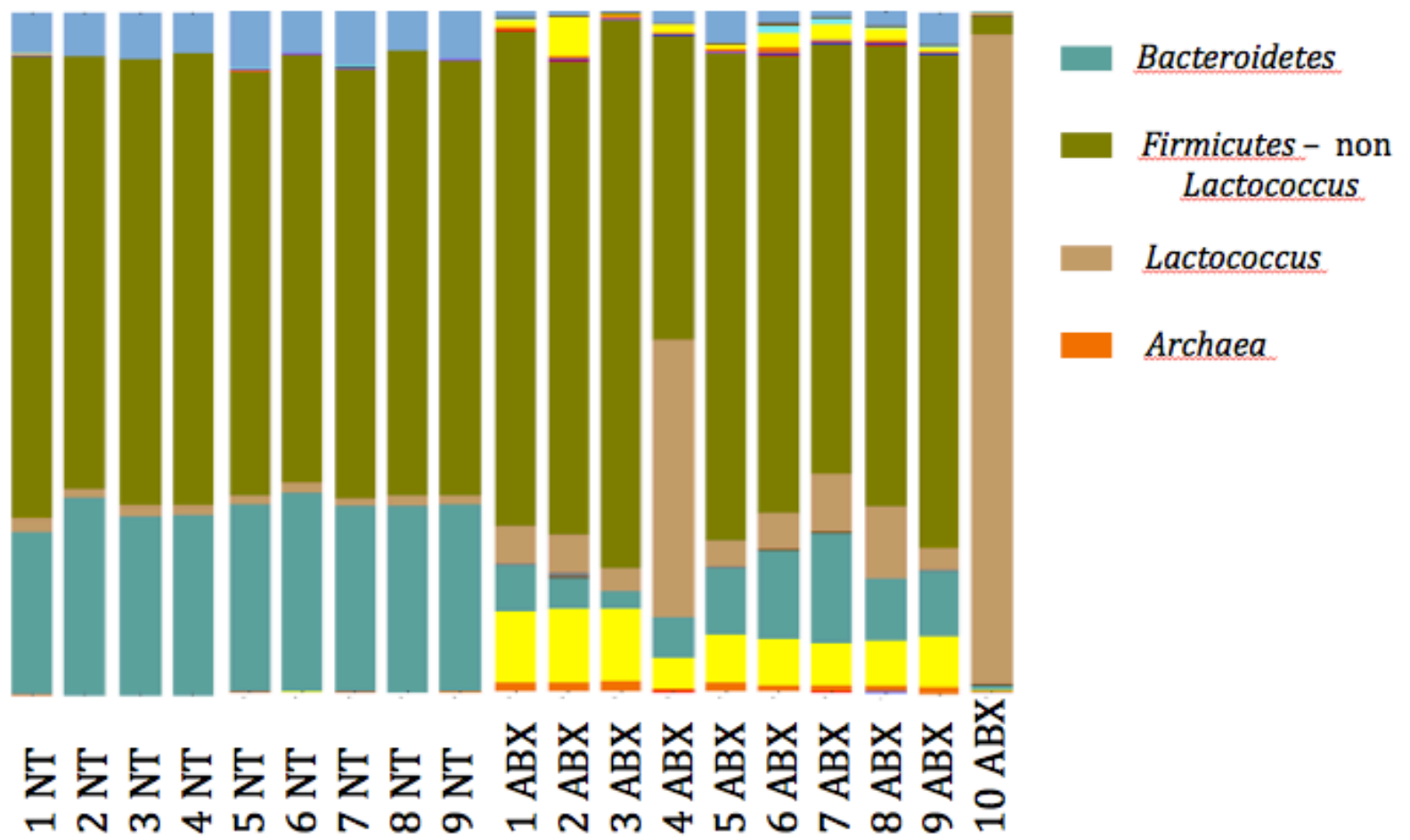

Figure 5. Taxonomic assignment of phylotypes found in feces from control and antibiotic-treated mice at the class level from $\mathrm{V} 4$ region 16S rRNA sequencing analysis . The total height of the bar represents $100 \%$ of the assigned sequences after quality filtering and the height of the individually colored bars represents the percentage of the class of organisms. The expansion of Lactococcus within the antibiotic-treated group is still present, but not near the percentages as seen with the V3 data (Fig 4.) A loss of Bacteroidetes was still observed, however a decrease in non-Lactococcus Firmicutes was not detected with the V4 sequencing data. 
A

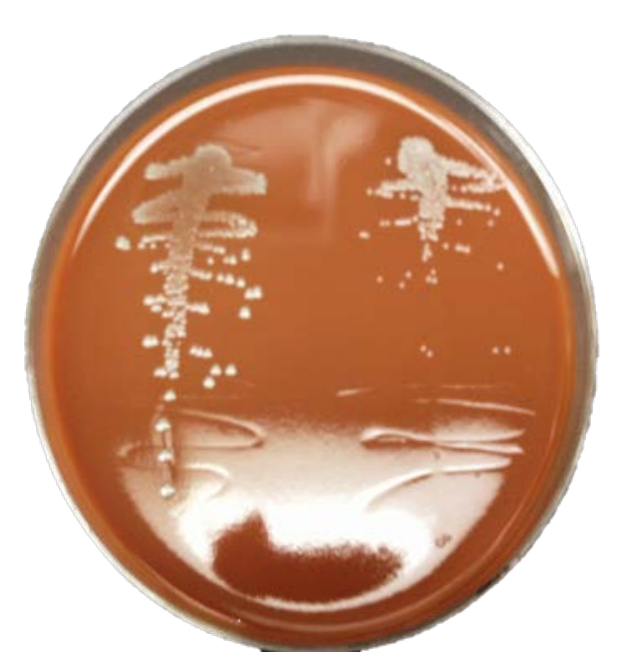

B

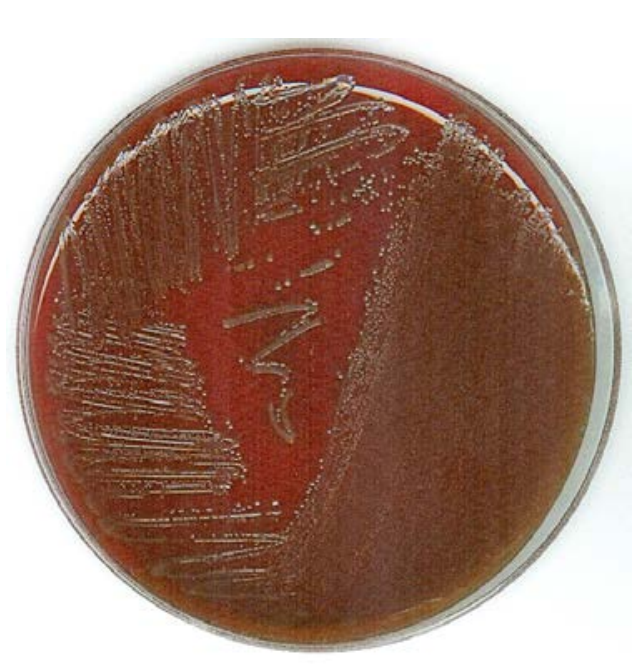

Figure 6. Cultures of feces from antibiotic-treated mice. Aerobic cultures of the fecal pellets from the antibiotic-treated mice yielded an abundant growth of yeast (A). Upon closer examination, there were pinpoint $\alpha$-hemolytic colonies that were subcultured for isolation on new $5 \%$ sheep blood agar plate (B). 
A

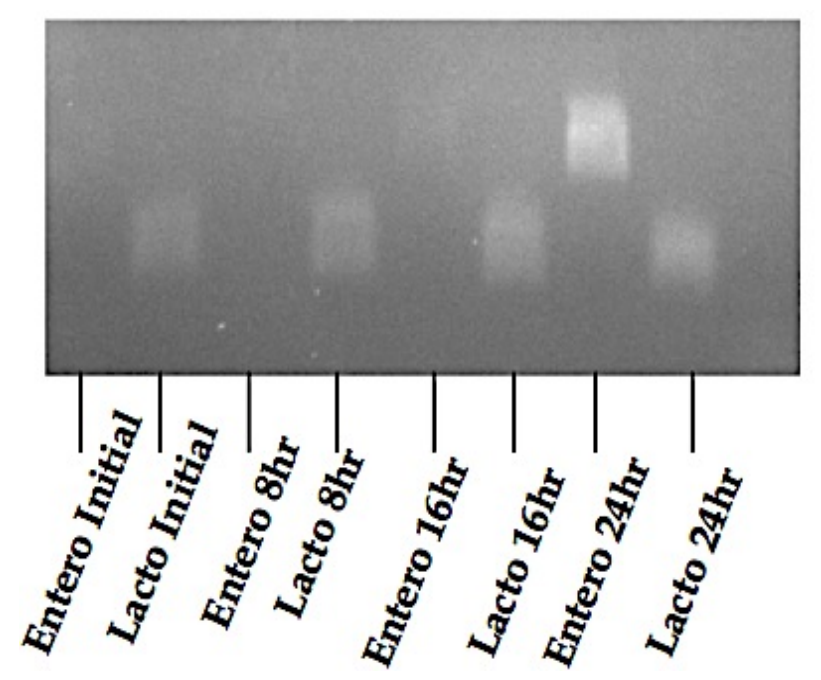

B

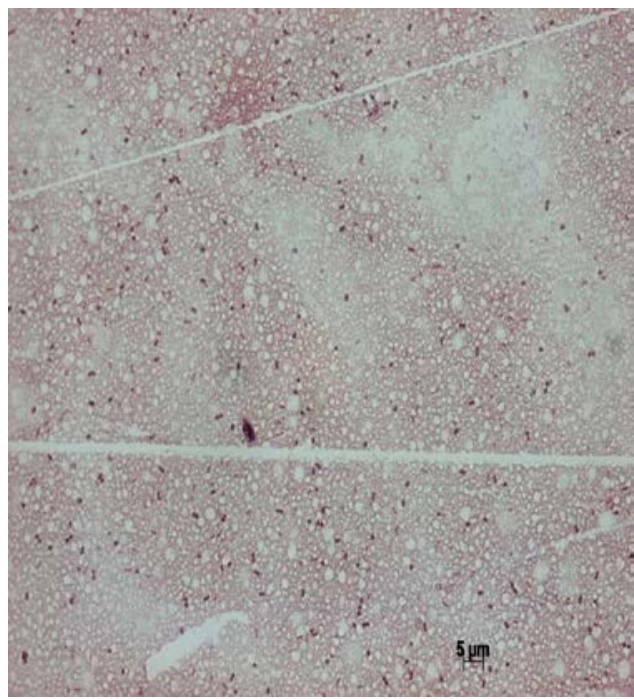

Figure 7. Culturing of mouse fecal pellets and growth time point assay. A 24-hour growth rate, time point assay was performed and the growth of Lactococcus and Enterococcus were compared in culturing in condensed milk. PCR amplification (A) was performed with bacteriaspecific primers at four separate time points; 0, 8 hours, 16 hours and 24 hour time points. Results indicate that the Lactococcus is present initially but never propagates, and Enterococcus is detected at 16 hours with an increase of PCR product at 24 hours. A Gram stain of the milk at 24 hours (B) displays Gram-positive cocci and yeast. 
A

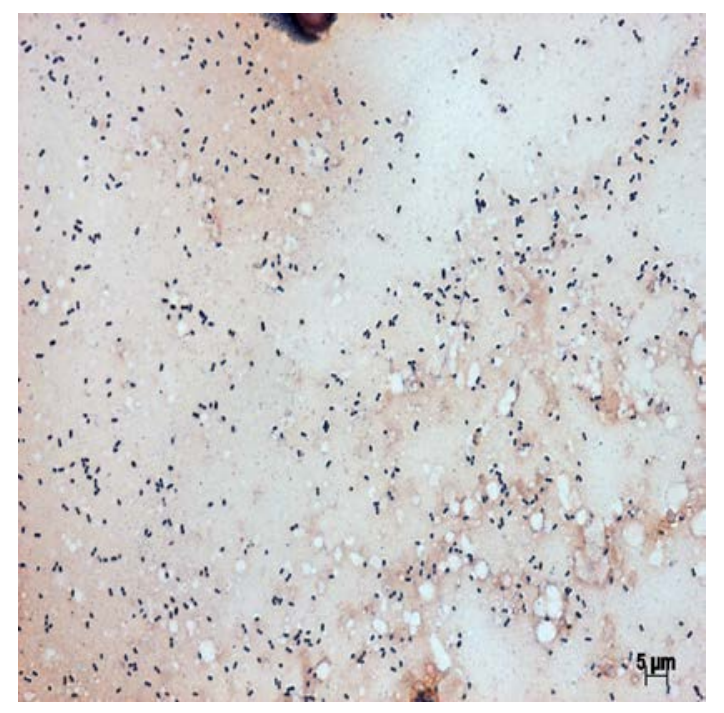

B

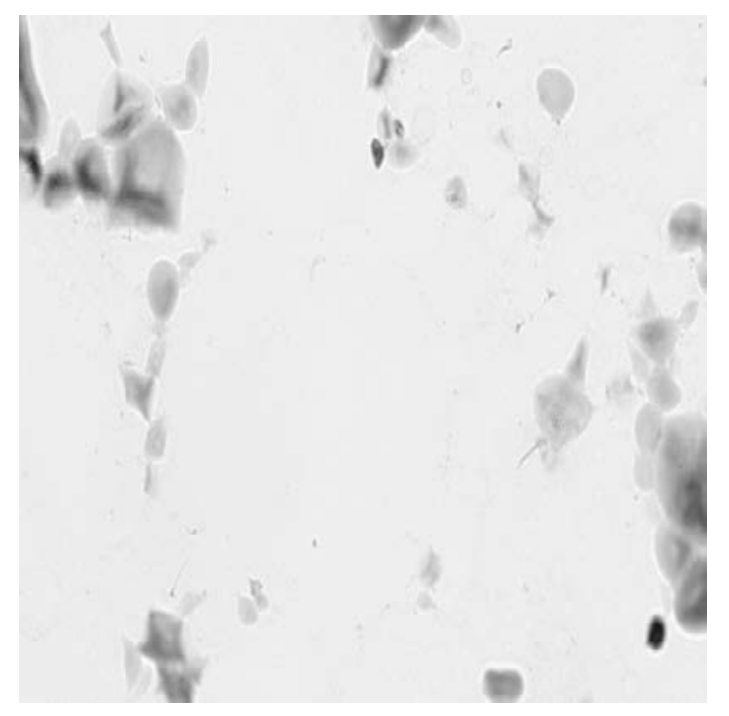

Figure 8. Gram stain analysis (A) of Open Source and 2018 Teklad Global 18\% Protein Rodent Diet chow (B). The Gram stain displays homogenous, Gram-positive coccobacilli in the Open Source diet that resembles the organism from the fecal samples, whereas no bacterial cells were observed in the 2018 Teklad Global chow. 

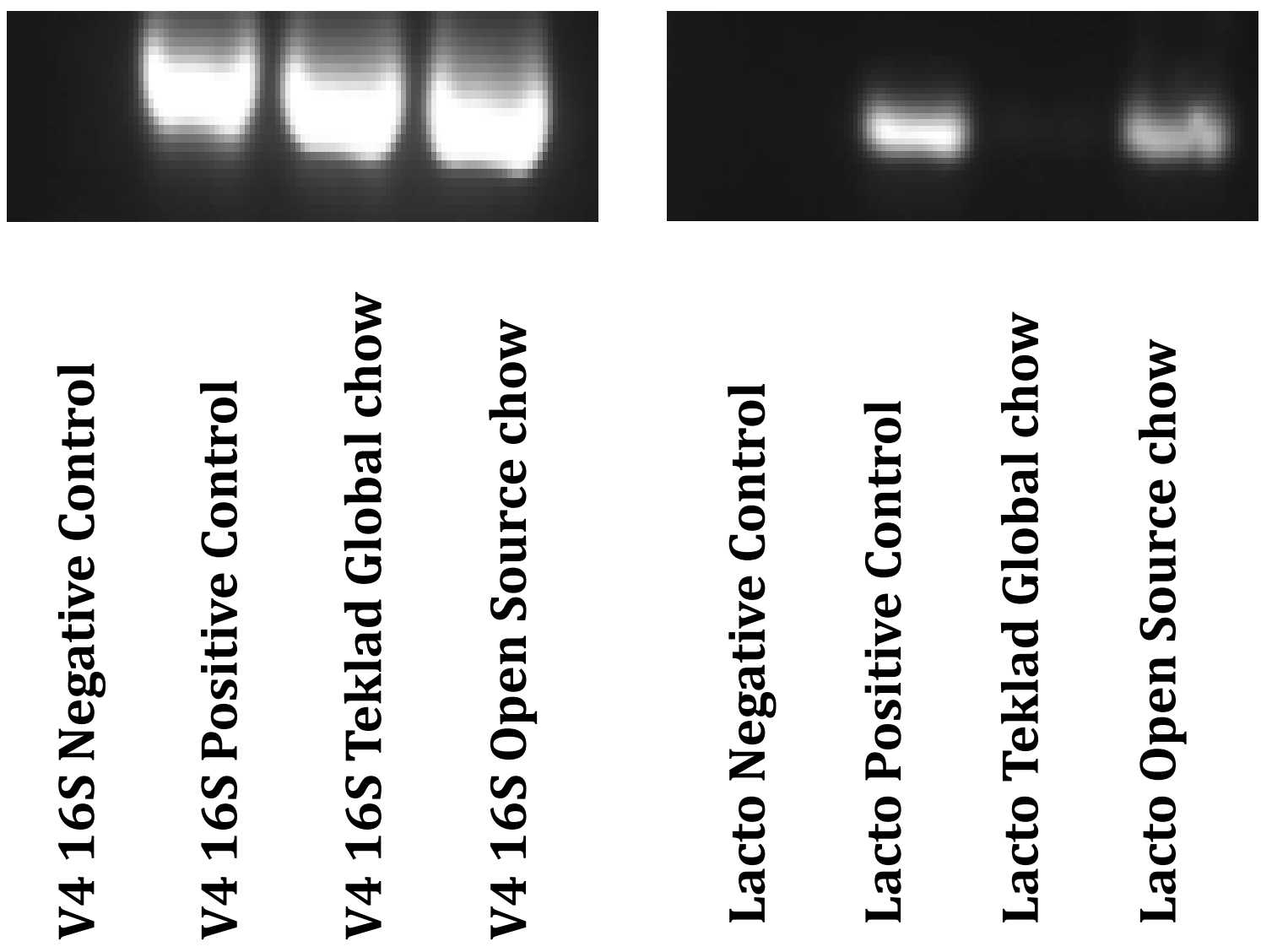

Figure 9. PCR analysis demonstrates Lactococcus DNA present in the Open Source Diet chow. The Open Source Diet chow and the 2018 Teklad Global chow were emulsified in sterile, distilled water and PCR reactions were performed for $16 \mathrm{~S}$ rRNA as well as Lactococcus specific primers. 
A

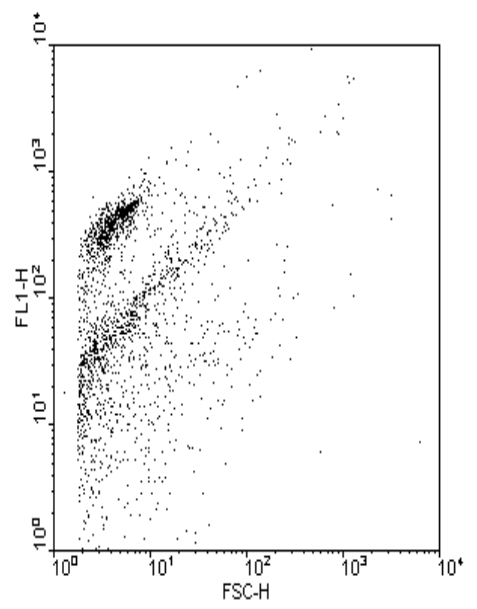

B

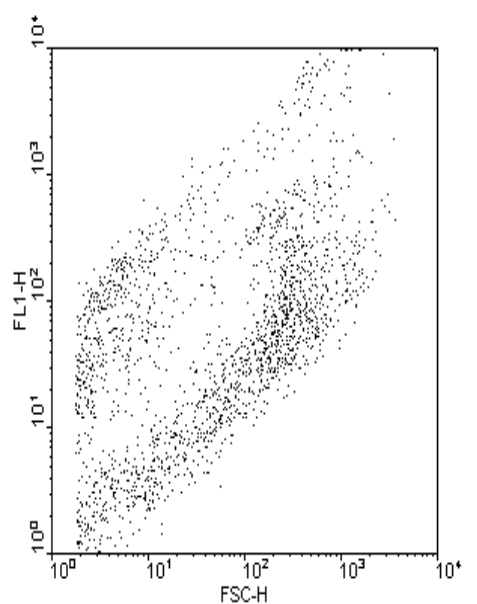

C

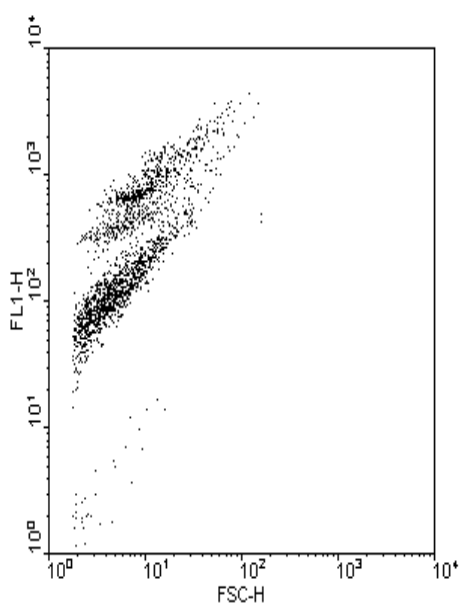

Figure 10. Flow cytometric analysis of chow. Open Source Diet (A) has a distinct bacterial population that resembles the fecal samples of the antibiotic-treated mice (see Fig 2B) and this population is absent in 2018 Teklad Global chow (B). An analysis on a purified culture of Lactococcus is shown in (C) for comparison. 
A

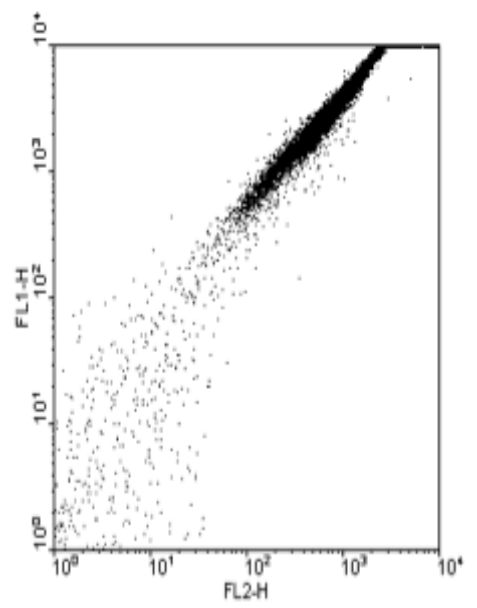

B

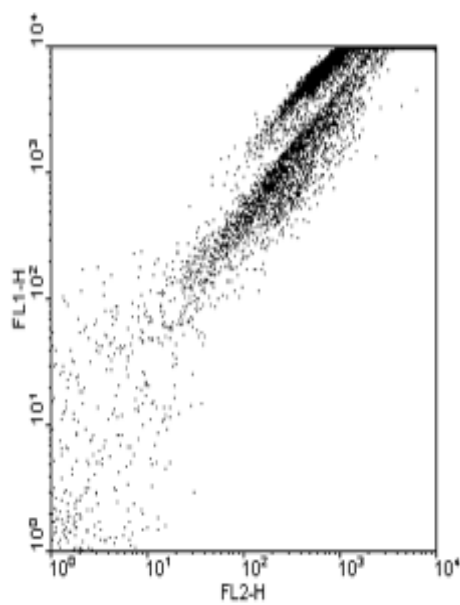

C

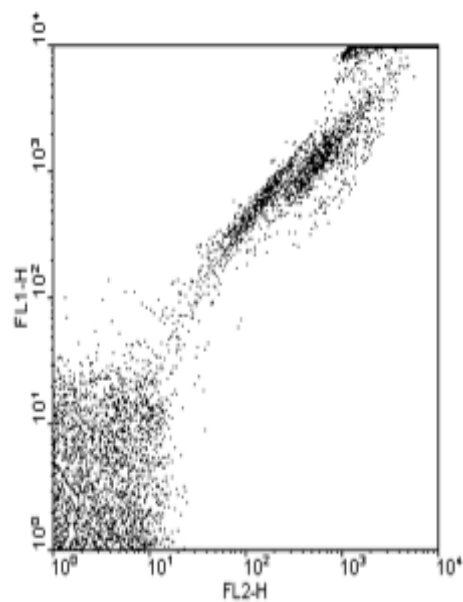

Figure 11. Viability analysis of chow via flow cytometry.

Invitrogen's Live/Dead kit for bacteria utilizes Syto9 and propidium iodide (PI) to elicit different staining characteristics between cells that have an uncompromised cellular wall versus cells that are non-viable and have perforations within the cell wall. If the cell wall has been compromised the PI will quench the Syto9 and present a homologous population, as seen in the E. coli cells fixed with $4 \%$ paraformaldehyde (A). A dual population will be seen in viable cells that maintain their cell walls, as seen in the sample of cultured Lactococcus (B). When the Open Source chow is examined (C) there is a loss of the dual population, identifying that the bacterial cell wall has been compromised and the cells are not viable. 


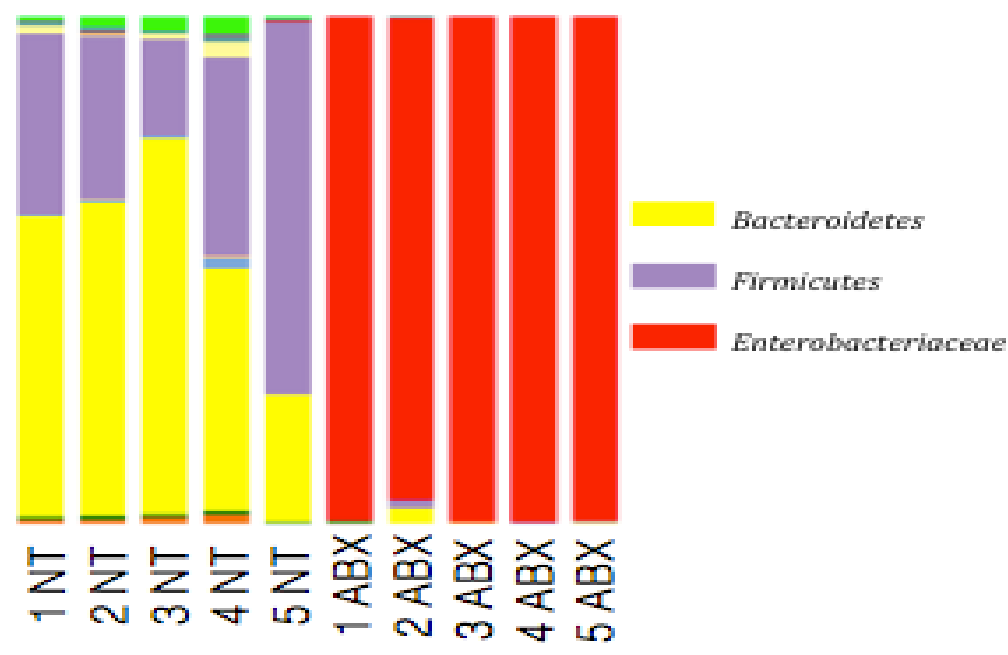

Figure 12. Taxonomic assignment of the GI microbiome after sequencing V3 region of $16 \mathrm{~S}$ rRNA sequencing from antibiotic-treated and control mice fed 2018 Teklad Global chow. The total height of the bar represents $100 \%$ of the assigned sequences and the height of the individually colored bars represents the percentage of the order of organisms. 

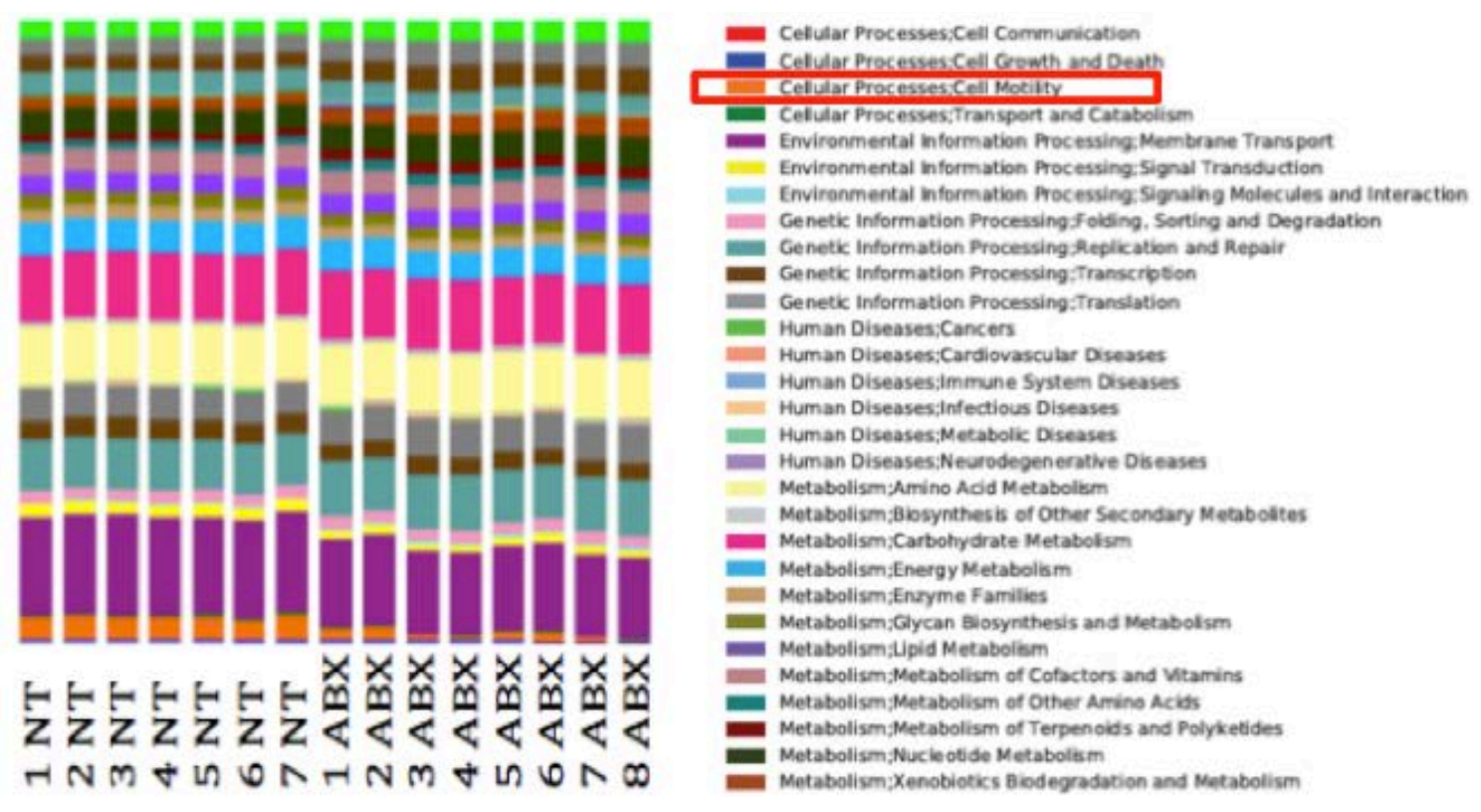

Figure 13. Metagenomic analysis. Predictive metagenomic functions were applied through PICRUSt bioinformatics software. The most notable change between the antibiotic-treated and control group was in motility, which correlates with Lactococcus being a non-motile organism. 
A

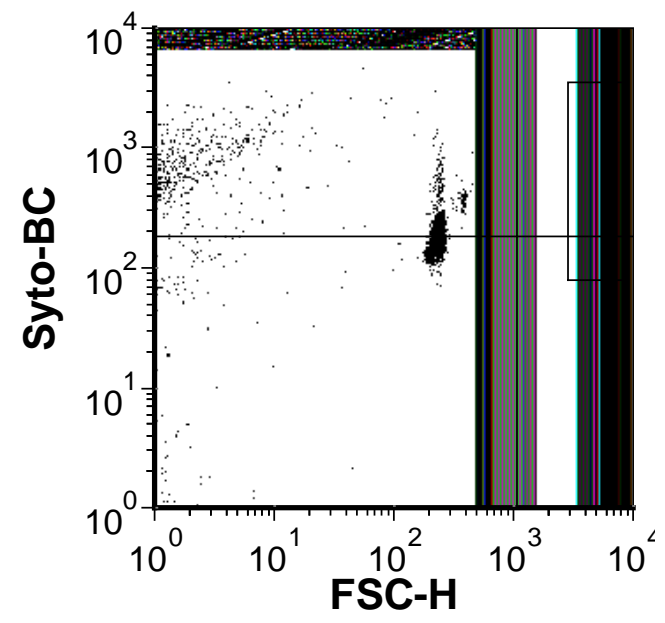

B

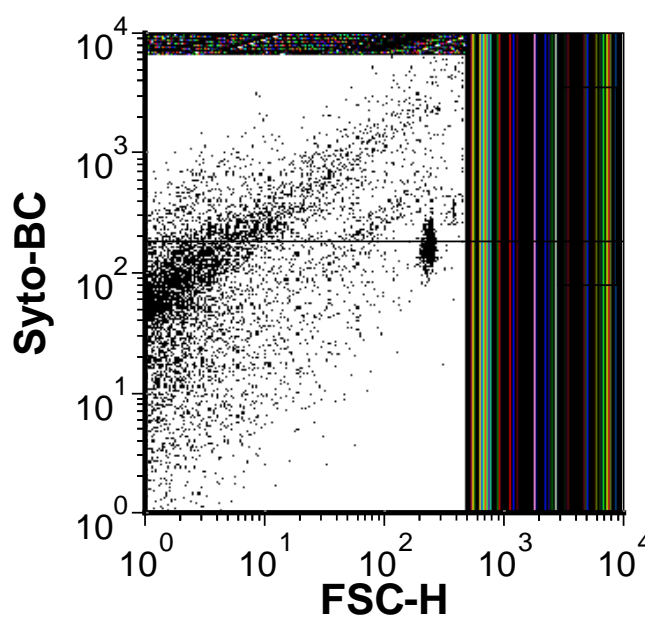

Figure 14. Quantitative flow cytometry performed on suspension from fecal pellets from control and antibiotic-treated mice on Teklad global chow. Samples from 3 mice in each group were analyzed and displayed an average of a $2-\log _{10}$ difference in bacterial load. The average cell count of the control group was $5.3 \times 10^{6}$ and the antibiotic-treated group was $5.9 \times 10^{4}$ respectively. A heterogeneous population of cells is displayed in the control sample (A), when compared to the antibiotic-treated sample (B). Of note, most of the cells counted in the antibiotic-treated sample (B) appear to be debris. 


\section{DISCUSSION}

Preliminary studies in this project were undertaken to examine the role of diet in affecting the intestinal microbiome; particularly in the context of antibiotic-induced perturbations in an intestinal ecosystem. We initially wanted to add quantitative analysis to previously reported studies that relied on culture methods ${ }^{[41]}$ or qPCR $^{[34]}$ to quantify perturbation of the $16 \mathrm{~S}$ gene in studies of the microbiome.

Quantitative flow cytometry revealed an average of a 1- $\log _{10}$ decrease in bacteria in the fecal samples from antibiotic-treated mice when compared to the control fecal samples. This result conflicts with

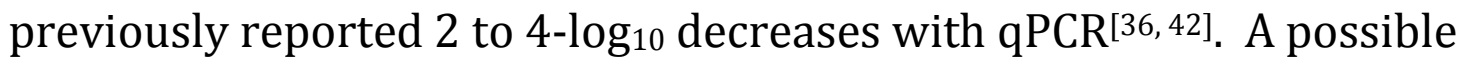
explanation for this disparity is variation in the number of $16 \mathrm{~S}$ rRNA gene copies that each bacterial organism possesses, the number of copies can vary from 1 to 15 with an average of 7.[43] Thus, if remaining bacteria have a disproportionately low copy number of $16 \mathrm{~S}$ gene then this could account for the discrepancy. 
Flow cytometric analysis of fecal suspensions from antibiotic-treated mice showed a homogenous population that was staining with the DNA stain SytoBC, and by forward scatter measurement were within the same parameters of known bacterial cells. Further, Gram stains of fecal suspensions from the antibiotic-treated mice yielded a morphologic picture dominated by Gram-positive coccobaccilli. When fecal suspensions from both antibiotic-treated and control samples were diluted to equal concentrations based on the flow cytometry data, the bacterial populations appeared equivalent in number by Gram stain in all observed fields. This result conflicted with the 2 to $4 \log _{10}$ difference that was previously reported. This was an unexpected finding given the type of antibiotics used in the system; the metronidazole should eliminate the anaerobic bacteria, neomycin is effective against Gramnegative bacteria, vancomycin is highly effective against Gram-positive organisms, and the ampicillin should eradicate the remaining bacteria due to it's broad spectrum capabilities. Multiple attempts at culturing the organism aerobically, anaerobically and microaerophically were essentially negative, with the exception of the growth of yeast (that was present in low numbers in the Gram stains) and very low numbers of other colonies, which conflicts with the Gram stain and flow cytometry 
data. The potential explanations included: 1) the Gram-positive coccobaccillus is a fastidious bacteria that was highly antibiotic resistant; 2) the organism was Archaea; 3) material identified as a microorganism by flow cytometer and Gram stain was actually debris; 4) the Gram-positive organism was dead.

The idea that the organism could be Archaea was provocative since Methanobrevibacter smithii is known to colonize the human gut [44], but to our knowledge it is unknown if they reside within the murine gut. However, PCR analysis of the fecal suspensions of the antibiotic-treated mice failed to amplify with Archaea specific primers (data not shown), and fluorescent staining for both peptidoglycan and DNA provided further evidence that the putative cells were bacteria.

PCR amplification was carried out at 30 cycles to ensure adequate concentrations could be recovered to generate the libraries. PCR reactions were performed with equivalent amounts of DNA quantified by nanodrop, however it is impossible to distinguish between mouse DNA and bacterial DNA in the measurement. 
High-throughput sequencing was performed on the fecal suspension to compare the gut microbiome from the antibiotic-treated mice to the control mice fed Open Source chow. Sequence analysis of both the V3 and V4 regions of the 16s rRNA gene indicated an expansion of Lactococcus. The increase in Lactococcus was more evident in analysis of the V3 region; however there was still an increase when the $\mathrm{V} 4$ region was analyzed from the antibiotic-treated samples as well. The presence of Lactococcus was confirmed by PCR amplification of fecal DNA with Lactococcus specific primers. Therefore, based on morphological determinants, high-throughput sequencing, and PCR performed with specific primers, we concluded that the organism present in the feces of the antibiotic-treated mice was Lactococcus. This result is consistent with the findings presented by Dollive et al.[36] However, Lactococcus is not a fastidious organism so it should have been readily cultivated, and although previous studies have identified vancomycin resistant strains of Lactococcus ${ }^{[3]}$ an ampicillin resistant strain has not yet been identified. 
The differences in the sequencing analysis between the V3 and V4 (Fig 4 \& 5) regions can be explained by the length of product, the length of the V3 product allowed us to overlap reads and create contigs, where the V4 region was too long and the sequence analysis was created from single strand reads. The numbers at the bottom of the graphs correlate with the mouse number and NT refers to the control group, no antibiotic treatment given and the $\mathrm{ABX}$ is the antibiotic-treated group. For both the V3 and V4 sequence runs the same number of samples were processed, however some of the samples failed to produce adequate reads due to various reasons.

Repeat culturing of the feces of the antibiotic-treated mice revealed scant, pinpoint $\alpha$-hemolytic colonies mixed in with the yeast, which we initially surmised was Lactococcus. Significant efforts to enrich this bacterium in Lactococcus-specific media (M17 agar supplemented with $10 \%$ sucrose and $0.5 \%$ glucose) were fruitless, and Sanger sequencing of the $16 \mathrm{~S}$ gene amplified from purified colonies identified the organism as Enterococcus casseliflavus, not Lactococcus. Reexamination of the sequences from the high-throughput sequencing data confirmed that a very small percentage of Enterococcus within the fecal suspensions of 
the antibiotic-treated mice was detected. Nevertheless, comparison of the previous V3 and V4 sequences in the high-throughput data that were identified as Lactococcus did not match the V3 or V4 region of the 16S gene from the Enterococcus isolates. Previous studies have reported that some species of Enterococcus have been shown to be resistant to vancomycin and ampicillin[53-55], and follow-up testing on the isolates from the fecal samples from the antibiotic-treated mice found the Enterococcus strain to be resistant to vancomycin and intermediate to ampicillin (data not shown). Thus we were forced to resolve the seemingly incongruous dilemma of whether the organism that is observed by Gram-stain and flow cytometry is Lactococcus or Enterococcus, and if it was Lactococcus why did it not grow in culture.

A previously published study identified a region within the bacterial elongation factor tufgene that was capable of differentiating between members of Enterococcus and Lactococcus. ${ }^{[49]}$ Therefore, tuf gene sequencing of DNA from fecal suspensions from antibiotic-treated mice was performed, and $100 \%$ yielded a match for Lactococcus. Now convinced that the organism in question was Lactococcus, we were still perplexed that it would not grow. Studies have shown that both 


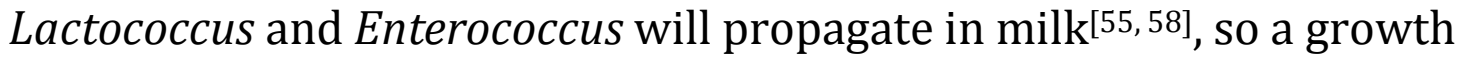
assay was established using the milk as a medium and feces from antibiotic-treated mice as the inoculum. It was determined that the Enterococcus was propagating in the milk, however Lactococcus DNA that was detected initially failed to produce an increase in intensity by PCR throughout 24 hours of culture. Thus, we concluded that the Lactococcus present in the feces was non-viable, but the source was still unknown.

The possibility that the Lactococcus was a contaminant in the Open Source chow prompted the examination of the chow. Gram stains revealed that the chow was contaminated with Gram-positive coccobaccilli, and PCR analysis confirmed the organism to be Lactococcus. Cultures of the food yielded no growth, so it was concluded that the bacteria were non-viable. A quantitative flow cytometric evaluation of the Open Source chow determined a bacterial count of $3 \times 10^{12}$ bacteria/g of chow. With an average daily consumption of $4.5 \mathrm{~g}$ of chow/per mouse, the mice were consuming an average of $1.4 \times 10^{13}$ non-viable Lactococcus per day. An average of $1.1 \times 10^{10}$ Lactococcus cells/g of feces was recovered from the antibiotic-treated mice, with an 
average of $\sim 100 \mathrm{mg}$ of feces occupying the intestines at any given time that would account for $10^{12}$ Lactococcus cells residing within the intestines. Our bacterial counts conflict with Dollive et al.[36] previously reported $5 \times 10^{4}$ bacteria/per fecal pellet; one potential explanation is that the DNA within the dead Lactococcus is degrading and lacks the stability to amplify by qPCR, however the SytoBC stain is still capable of binding to the DNA making detection by the flow cytometer possible. Given this scenario, the dead Lactococcus could be though of as an empty peanut shell that facilitates transport of its degrading DNA throughout the intestinal tract. Interestingly, we discovered proportional levels of Lactococcus present throughout the intestinal tract, to include the small intestines just above the ilealcecal junction, which is normally has a much lower concentration of microorganisms at an average of $10^{4}$ bacterial cells / $\mathrm{g}^{[57]}$.

Lactococcus is widely used in the dairy industry because of its homofermentor capabilities[58]; it solely produces lactic acid as it metabolizes glucose. Lactococcus acidifies milk rapidly and prevents the overgrowth of other bacteria that would cause spoilage ${ }^{[58]}$. One of the main sources of protein in the Open Source chow is casein, which is 
derived from mammalian milk. It is quite possible that the Lactococcus in the chow is from the acidified milk that is used to introduce casein into the finalized pelletized chow. Casein is also present in Open Source Hi-fat diets that are commonly used in nutritional studies, which explains results from earlier studies with the presence of Lactococcuslike bacteria in fecal samples from antibiotic-treated mice fed a high-fat diet (Cuff et al. unpublished).

After the discovery of the Lactococcus in the Open Source chow we wanted to repeat the microbiome analysis of the $\mathrm{V} 3$ region with the mice on another chow. Teklad Global 2018 is the normal chow used in the WVU Health Sciences vivarium, and was confirmed to be negative for Lactococcus. Firmicutes and Bacteroidetes represented the majority of the bacterial population in the control mice samples that were fed Open Source chow; however with the Teklad Global chow there was a larger percentage of Bacteroidetes and a smaller percent of Firmicutes than with the Open Source chow. These data reflect the removal of the Lactococcus (which is in the Firmicute order) from the chow. The antibiotic-treated samples had a $>95 \%$ population of a lactose fermenting Enterobacteraceae that was identified as Escherichia coli by 
Vitek analysis performed in the laboratory at Ruby Memorial hospital, Morgantown, WV. Our findings coincide with Hill et al.[42], in respect to an increase in Enterobacteraceae in mice that are treated with antibiotics administered through drinking water, and no Lactococcus discovered when diet did not contain casein.

The antibiotic-treated fecal samples also showed a loss of bacterial motility genes when predictive metagenomic analysis was performed through PICRUSt, which is consistent with an increase in Lactococcus, which is non-motile. Although in minuscule percentages, Archaea was identified by sequencing the $\mathrm{V} 4$ region of $16 \mathrm{~S}$ rRNA in the antibiotictreated mice.

Interestingly, there was no yeast recovered from the feces of the antibiotic-treated mice that were fed the Teklad Global even after 10 wks of treatment. Another group of antibiotic-treated mice that were fed the Teklad Global for 3 wks and were then converted to the Open Source chow for the remaining $7 \mathrm{wks}$ also failed to produce yeast in the feces. However, yeast was recovered at day 7 of antibiotic treatment in the feces of mice that were fed only the Open Source. Speculation as to 
why the mice that were fed the Teklad Global chow failed to grow yeast from the feces is that the Teklad Global lacked the sucrose supplementation that was included in the Open Source chow. The Open Source chow consists of $35 \%$ sucrose, which yeast utilize as a source of nutrients, especially Candida spp. ${ }^{[52]}$ In the mice that were fed solely the Open Source chow, we speculate that the addition of the sucrose supported the growth of Candida and outcompeted antibiotic-resistant bacteria from colonizing. We further speculate that the intestines within the mice fed the Teklad Global chow from the beginning supported the antibiotic resistant bacteria the opportunity to colonize and flourish to outcompete the yeast once the Open Source chow was introduced. An experimental approach to check if the dead Lactococcus was promoting the growth of the yeast would be to fortify the Teklad Global chow with dead Lactococcus and feed it to the mice.

Our results demonstrate that ingestion of chow contaminated with nonviable Lactococcus is capable of passage through the intestinal tract and be displayed as the dominant bacteria within the feces in this model of antibiotic-ablation of the intestinal microbiome. It is possible for bacteria present in food to modulate intestinal microbiome studies. The 
results of our study have paved the way for potential future studies of whether and/or to what extent food-derived, non-viable bacteria can alter intestinal physiology. 


\section{REFERENCES}

1. Kayama $\mathrm{H}$, et al. Regulation of intestinal homeostasis by innate and adaptive immunity. International Immunology. 2012: dxs094v1-dxs094.

2. Guarner F, et al. (2003). Gut flora in health and disease. The Lancet 361. 2003; (9356): 512-9. doi:10.1016/S0140-6736(03)12489-0.

3. Erejuwa 0, et al. Modulation of gut microbiota in the management of metabolic disorders: the prospects and challenges. International Journal of Molecular Sciences. 2014, 15, 4158-4188; doi:10.3390/ijms15034158.

4. Burkholder P, et al. Synthesis of vitamins by intestinal bacteria. PNAS. 1942; 28(7): 285-289.

5. Jeffery I, et al. Diet-microbiota interactions and their implications for healthy living. Nutrients. 2013, 5, 234-252; doi:10.3390/nu5010234.

6. Savage, D. C. Microbial ecology of the gastrointestinal tract. Annual Review of Microbiology. 1977: 31; 107-33. doi:10.1146/annurev.mi.31.100177.000543.

7. Berg, R. The indigenous gastrointestinal microflora. Trends in Microbiology. 1996: 4 (11): 430-5. doi:10.1016/0966-842X(96)10057-3.

8. Willey J, et al.; Prescott's Microbiology (8th ed.). New York: McGraw Hill. pp. 731737. ISBN 9780077350130.

9. Backhed F, et al. Host-bacterial mutualism in the human intestine. Science 307, 1915 (2005);DOI: 10.1126/science.1104816

10. Turnbaugh P.J., et al. The human microbiome project. Nature. 2007: 449: 804810. doi:10.1038/nature06244

11. Ley R.E., et al. Ecological and evolutionary forces shaping microbial diversity in the human intestine. Cell. 2006: 124: 837-848. doi:10.1016/j.cell.2006.02.017

12. Peterson J, et al. The NIH human microbiome project. Genome Res. 2009; 19(12):2317-2323. [PubMed: 19819907]

13. Structure, function and diversity of the healthy human microbiome. Nature. 2012; 486(7402):207-214. [PubMed: 22699609]

14. Reyes A, et al. Viruses in the faecal microbiota of monozygotic twins and their mothers. Nature. 2010; 466(7304):334-338. [PubMed: 20631792] 
15. O'Hara A, et al. The gut flora as a forgotten organ. EMBO Reports 2006; 7: 688693.

16. Ottman N, The function of our microbiota: who is out there and what do they do? Frontiers in Cellular and Infection Microbiology 2012. August: doi: 10.3389/fcimb.2012.00104.

17. Biasucci G, et al. Caesaren deliver may affect the early biodiversity of intestinal bacteria. Journal of Nutrition 2008: 138; 1796S-1800S.

18. Bezirtzoglou E, et al. Microbiota profile in feces of breast-and formula-fed newborns by using fluorescence in situ hybridization (FISH). Anaerobe 2011: 17, 478-482.

19. Koenig J, et al. Succession of microbial consortia in the developing infant gut microbiome. PNAS: 108(Suppl. 1); 4578-4585.

20. Biagi E, et al. Through ageing, and beyond: gut microbiota and inflammatory status in seniors and centenarians. PLoS ONE 2010: 5:e10667. doi: 10.137/journal.pone.0010667.

21. Witman WB, et al. Prokaryotes: the unseen majority. Proc Natl Acad Sci USA 1998; 95:6578-6583 [PubMed: 17183309].

22. Hildebrandt, et al. High-fat diet determines the composition of the murine gut microbiome independently of obesity. Gastroenterology 2009; 137:1716-1724.

23. Hugenholz, $P$, et al. Impact of culture-independent studies on the emerging phylogenetic view of bacterial diversity. J. Bacteriol 1998; 180 (18): 4765-74.

24. De Filippo C, et al. Impact of diet in shaping gut microbiota revealed by a comparative study in children from Europe and rural Africa. PNAS 2010, 107:14691-14696.

25. Hopkins MJ, et al. Age and disease related changes in intestinal bacterial populations assessed by cell culture, $16 \mathrm{~S}$ rRNA abundance, and community cellular fatty acid profiles. Gut 2001, 48:198-205.

26. Khachatryan ZA, et al. Predominant role of host genetics in controlling the composition of gut microbiota. PLoS ONE 2008, 3:e3064.

27. Turnbaugh PJ, et al. The effect of diet on the human gut microbiome: a metagenomic analysis in humanized gnotobiotic mice. Sci Transl Med 2009, 1:6ra14ra.

28. Turnbaugh PJ, et al. Organismal, genetic, and transcriptional variation in the 
deeply sequenced gut microbiomes of identical twins. PNAS 2010, 107:7503-7508.

29. Yatsunenko T, et al. Human gut microbiome viewed across age and geography. Nature 2012, 486:222-227.

30. Sheddy SA, et al. Opportunities and challenges for gut microbiota studies in the Indian population. Microbiome 2013, 1:24.

31. Hooper LV, et al. Commensal host-bacterial relationships in the gut. Science. 2001, May 11; 292(5519): 1115-8.

32. Zackular J, et al. The gut microbiome modulates colon tumorigenesis. mBIO 2013; 4(6): doi: 10.1128/mBio.00692-13.

33. Fouhy F, et al. High-throughput sequencing reveals the incomplete, short-term recovery of infant gut microbiota following parentaeral antibiotic treatment with ampicillin and gentamicin. Antimicrobial Agents and Chemotherapy 2012: 56(11); doi:10.1128/AAC00789-12.

34. Buffie G, et al. Microbiota-mediated colonization resistance against intestinal pathogens. Nature Reviews Immunology 13, 790-801 (2013); doi:10.1038/nri3535.

35. Asahara T, et al. Protective effect of Lactobacillus casei strain shirota against lethal infection with multi-drug resistant Salmonella enterica serovar typhurium DT104 in mice. Journal of Applied Microbiology 2010: 110, 163-173.

36. Dollive S., et al. Fungi of the murine gut: episodic variation and proliferation during antibiotic treatment. PLOS One. 2013; 8(8): (e71806).

37. Sekirov I, et al. Antibiotic-induced perturbations of the intestinal microbiota alter host susceptibility to enteric infection. Infect Immun. 2008;76(10):4726-4736.

38. Antonopoulos DA, et al. Reproducible community dynamics of the gastrointestinal microbiota following antibiotic perturbation. Infect Immun. 2009;77(6):2367-2375. doi: 10.1128/IAI.01520-08.

39. Croswell A, et al. Prolonged impact of antibiotics on intestinal microbial ecology and susceptibility to enteric Salmonella infection. Infect Immun. 2009;77(7):27412753. doi: 10.1128/IAI.00006-09.

40. Garner CD, et al. Perturbation of the small intestine microbial ecology by streptomycin alters pathology in a Salmonella enterica serovar typhimurium murine model of infection. Infect Immun. 2009;77(7):2691-2702.

41. Lawley TD, et al. Antibiotic treatment of clostridium difficile carrier mice triggers a supershedder state, spore-mediated transmission, and severe disease in 
immunocompromised hosts. Infect Immun. 2009;77(9):3661-3669.

42. Hill D.A., et al. Metagenomic analyses reveal antibiotic-induced temporal and spatial changes in intestinal microbiota with associated alterations in immune cell homeostasis. Mucosal Immunology. 2010; 3(2): 148-158. doi:10.1038/mi.2009.132.

43. Reeves A, et al. Suppression of Clostridium difficile in the gastrointestinal tracts of germfree mice inoculated with a murine isolate from the family Lachnospiraceae. Infectious Immunology 2012: 80(11) 3786: doi:01.1128/IAI.00647-12.

44. van Nood E, et al. Duodenal infusion of donor feces for recurrent clostridium difficile The New England Journal of Medicine 2013: 368(5); doi:10.1056/NEJMoa1205037.

45. Duncan SH, et al. Cultivable bacterial diversity from the human colon. Letters in Applied Microbiology 2007. 44(4): 343-350. Doi:10.1111/j.1472765X.2007.02129.x.

46. Goodman A., et al. Extensive personal human gut microbiota culture collections characterized and manipulated in gnotobiotic mice. PNAS 2011; 108(15): 62526257. doi:10.1073/pnas.1102938108.

47. Bartram A, et al. Generation of multimillion-sequence $16 \mathrm{~S}$ rRNA gene libraries from complex microbial communities by assembling paired-end illumina reads. Applied and Environmental Microbiology, June 2011; 3846-3852: doi:10.1128/AEM.02772-10.

48. Caparoso JG, et al. Global patterns of $16 \mathrm{~S}$ rRNA diversity at a depth of millions of sequences per sampe. PNAS 2011: 108; doi:10.1073/pnas.1000080107.

49. Li X., et al. Use of tuf as a target for sequence-based identification of Grampositive cocci of the genus Enterococcus, Streptococcus, coagulase-negative Staphylococcus, and Lactococcus. Annals of Clinical Microbiology and Antimicrobials. 2012, 11:31. http://www.ann-clinmicrob.com/content/11/1/31

50. Langille MG, et al. Predictive functional profiling of microbial communities using 16S rRNA marker gene sequences. Nature Biotechnology 2013; 31; 814-821: doi:10.1038/nbt.2676.

51. Kembel S.W., et al. Incorporating $16 \mathrm{~S}$ gene copy number information improves estimates of microbial diversity and abundance. PLOS Computational Biology. 2012; 8 (10). doi: 10.1371/journal.pcbi.1002743.g001.

52. Hoffmann C., et al. Archaea and fungi of the human gut microbiome: correlations with diet and bacterial residents. PLoS ONE 2013; 8(6): e66019. doi:10.1371/journal/pone0066019. 
53. Wagner D., Vancomycin-resistant Lactococcus lactis $1 \mathrm{~A}-1$ isolated from a competitive exclusion product transfers vancomycin resistance genes to Staphylocccous aureus. The Open Food Journal, 2008: 2; 72-76.

54. Schouten M.A., et al. Antimicrobial susceptibility patterns of enterococci causing infections in Europe. Journal of Antimicrobial Chemotherapy. 2003: 51 (2): 323331. doi: $10.1093 / \mathrm{jac} / \mathrm{dkg} 052$.

55. Garg S., et al. Enterococci in milk and milk products. Critical Reviews in Microbiology. 1991: 18(1): 15-45. doi: 10.3109/10408419109113508.

56. Jong A, et al. Transcriptional and gene regulatory network of Lactococcus lactis mg1363 during growth in milk. PLoS ONE. 2013: 8(1): e53085. doi: 10.1371/journal.pone.0053085.

57. Quigley E., et al. Small intestinal bacterial overgrowth: roles of antibiotics, prebiotics, and probiotics. Gastroenterology 2006; 130 (2 Suppl 1): S78-90. doi:10.1053/j.gastro.2005.11.046

58. James M. Modern Food Microbiology, 4th ed. Van Nostrand Reinhold, 1992

59. De Vos W.M., et al. Genetics and biotechnology of lactic acid bacteria. eds. Gasson, M.J. de Vos, W.M. Gene Cloning and Expression Systems in Lactococci. Blackie Academic \& Professional, 1994. pg. 52 - 53.

60. Santana I.L., et al. Dietary carbohydrates modulate Candida albicans development on the denture surface. PLoS ONE. 2013; 8(5): e64645. doi:10.1371/journal.pone.0064645

61. Pu Z.Y., et al. Integrated polymerase chain reaction-based procedures for the detection and identification of species and subspecies of the Gram-positive bacterial genus Lactococcus. Journal of Applied Microbiology. 2002; 93: 353-361.

62. Danbing K., et al. Development of a PCR assay for rapid detection of Enterococci. Journal of Clinical Microbiology. 1999; 37(11): 3497-3503. 